\title{
A convergent numerical scheme for integrodifferential kinetic models of angiogenesis
}

\author{
Luis L. Bonilla \\ Gregorio Millán Institute for Fluid Dynamics, Nanoscience and Industrial Mathematics, \\ Universidad Carlos III de Madrid, 28911 Leganés, Spain \\ Ana Carpio ${ }^{1}$ \\ Departamento de Matematica Aplicada, Universidad Complutense, 28040 Madrid, Spain, \\ tel:+34-91-3944407, fax:+34-91-3944607 \\ Manuel Carretero \\ Gregorio Millán Institute for Fluid Dynamics, Nanoscience and Industrial Mathematics, \\ Universidad Carlos III de Madrid, 28911 Leganés, Spain \\ Gema Duro \\ Departamento de Análisis Económico: Economía Cuantitativa, Universidad Autónoma de \\ Madrid, 28049 Madrid, Spain \\ Mihaela Negreanu \\ Departamento de Matematica Aplicada, Universidad Complutense, 28040 Madrid, Spain \\ Filippo Terragni \\ Gregorio Millán Institute for Fluid Dynamics, Nanoscience and Industrial Mathematics, \\ Universidad Carlos III de Madrid, 28911 Leganés, Spain
}

\begin{abstract}
We study a robust finite difference scheme for integrodifferential kinetic systems of Fokker-Planck type modeling tumor driven blood vessel growth. The scheme is of order one and enjoys positivity features. We analyze stability and convergence properties, and show that soliton-like asymptotic solutions are correctly captured. We also find good agreement with the solution of the original stochastic model from which the deterministic kinetic equations are derived working with ensemble averages. A numerical study clarifies the influence of velocity cut-offs on the solutions for exponentially decaying data.
\end{abstract}

Email addresses: bonilla@ing.uc3m.es (Luis L. Bonilla), carpio@mat.ucm.es (Ana Carpio ), manili@math.uc3m.es (Manuel Carretero), gema.duro@uam.es (Gema Duro), negreanu@mat.ucm.es (Mihaela Negreanu), fterragn@ing.uc3m.es (Filippo Terragni)

${ }^{1}$ Corresponding author

Preprint submitted to Elsevier

September 7, 2018 
Keywords: Kinetic model, Fokker-Planck, integrodifferential, angiogenesis.

\section{Introduction}

The process through which new blood vessels sprout from existing ones is called angiogenesis $[11,23]$. While blood vessel development is crucial for healthy organ growth and damaged tissue repair, it may also be prompted by tumors to support their spread [40] or cause immune and inflammatory diseases. In tumor induced angiogenic processes, high cell density lowers the oxygen and nutrient levels in the inner regions of the tumor. Cells release a substance, the tumor angiogenic factor, which diffuses reaching adjacent blood vessels. This substance triggers the emergence and spread of new vessel tips towards the tumor to supply the necrotic cells with additional resources, see Fig. 1. In an attempt to forbid tumor growth, some therapies aim to block blood vessel expansion [12, 23, 34, 45]. A wide literature on models focussing on different features of the angiogenesis process is available, see [27, 30, 32, 35, 39] for instance. Nevertheless, novel mathematical descriptions are continuously motivated by the appearance of new experimental observations. The deterministic kinetic model we consider here reproduces the stochastic nature of the blood vessel branching process. From a theoretical point of view, it is derived from

stochastic descriptions of the development of blood vessel networks by means of ensemble averages [42]. Kinetic models allow us to gain understanding on the evolution of the blood vessel network by means of asymptotic and numerical solutions. The ultimate goal would be to control the angiogenesis process to starve the tumor. Employing reliable numerical discretizations is essential in these studies. However, the presence of nonlocal terms in time and velocity in the kinetic model, as well as the nonlinear coupling with a diffusion problem, render this task quite challenging.

Let us detail the model structure next. We denote by $p$ and $C$ the density of actively moving blood vessel tips and the concentration of tumor angiogenic factor released by tumor cells, respectively. Their time evolution is governed by the following system of nondimensional equations $[3,4,5]$ :

$$
\begin{aligned}
\frac{\partial}{\partial t} p(\mathbf{x}, \mathbf{v}, t)= & \alpha(C(\mathbf{x}, t)) \delta_{\sigma_{v}}\left(\mathbf{v}-\mathbf{v}^{0}\right) p(\mathbf{x}, \mathbf{v}, t)-\Gamma p(\mathbf{x}, \mathbf{v}, t) \int_{0}^{t} d s \int d \mathbf{v}^{\prime} p\left(\mathbf{x}, \mathbf{v}^{\prime}, s\right) \\
& -\mathbf{v} \cdot \nabla_{\mathbf{x}} p(\mathbf{x}, \mathbf{v}, t)+\beta \operatorname{div}_{\mathbf{v}}(\mathbf{v} p(\mathbf{x}, \mathbf{v}, t))+ \\
& -\operatorname{div}_{\mathbf{v}}[\beta \mathbf{F}(C(\mathbf{x}, t)) p(\mathbf{x}, \mathbf{v}, t)]+\frac{\beta}{2} \Delta_{\mathbf{v}} p(\mathbf{x}, \mathbf{v}, t) \\
\frac{\partial}{\partial t} C(\mathbf{x}, t)= & \kappa \Delta_{\mathbf{x}} C(\mathbf{x}, t)-\chi C(\mathbf{x}, t) j(\mathbf{x}, t) \\
p(\mathbf{x}, \mathbf{v}, 0)= & p_{0}(\mathbf{x}, \mathbf{v}), \quad C(\mathbf{x}, 0)=C_{0}(\mathbf{x})
\end{aligned}
$$




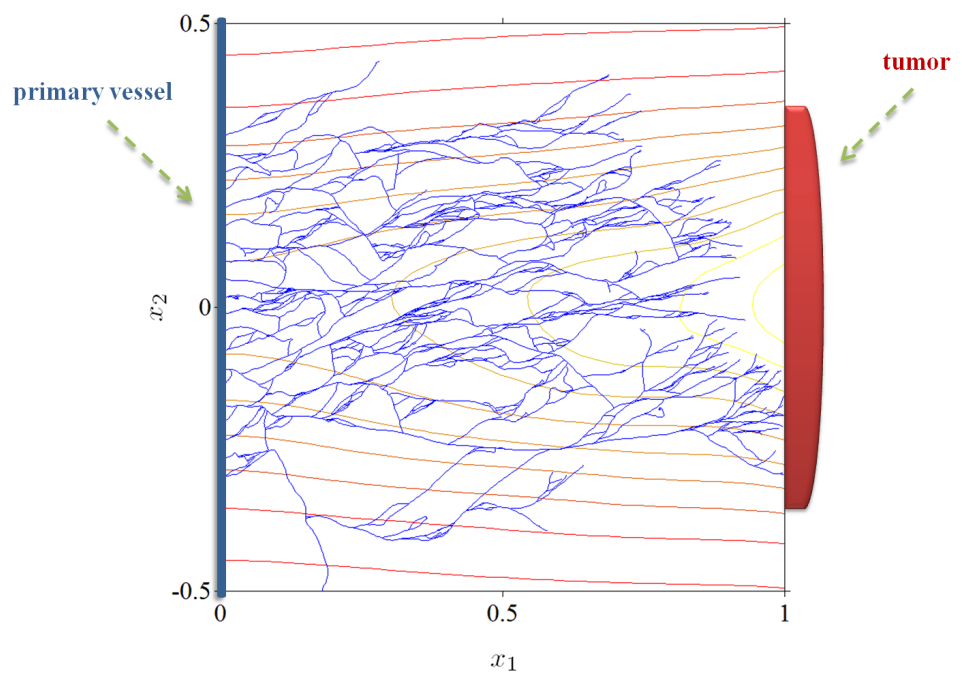

Figure 1: Network of blood vessels simulated by a stochastic model of tumor induced angiogenesis [4]. The level curves of the density of the tumor angiogenic factor (vessel endothelial growth factor) are also depicted.

where

$$
\begin{gathered}
\alpha(C(\mathbf{x}, t))=A \frac{C(\mathbf{x}, t)}{1+C(\mathbf{x}, t)}, \quad \mathbf{F}(C(\mathbf{x}, t))=\frac{\delta_{1}}{\left(1+\Gamma_{1} C(\mathbf{x}, t)\right)^{q_{1}}} \nabla_{\mathbf{x}} C(\mathbf{x}, t), \\
j(\mathbf{x}, t)=\int_{\mathbb{R}^{N}} \frac{|\mathbf{v}|}{1+e^{\left(\left|\mathbf{v}-\mathbf{v}^{0}\right|^{2}-\eta\right) / \epsilon}} p(\mathbf{x}, \mathbf{v}, t) d \mathbf{v}, \quad \rho(\mathbf{x}, t)=\int_{\mathbb{R}^{N}} p(\mathbf{x}, \mathbf{v}, t) d \mathbf{v},
\end{gathered}
$$

for $\mathbf{x} \in \Omega \subset \mathbb{R}^{N}, \mathbf{v} \in \mathbb{R}^{N}, N=2,3, t \in[0, \infty)$. The dimensionless parameters $\beta$, $\Gamma, \kappa, \chi, A, \Gamma_{1}, \delta_{1}, \eta, \epsilon$ and $q_{1}$ are positive. Typical values of these parameters are listed in Table 1. In dimension two, these models may be adapted to describe angiogenesis problems causing retinopathies [9]. We consider here the tumor environment. The integral sink $-\Gamma p \int_{0}^{t} \rho(\mathbf{x}, s) d s$ (in which $\rho$ defined in (5) is the marginal tip density) expresses that a vessel tip ceases to be active when it encounters another vessel because it joins the other vessel through a biological process called anastomosis. The anastomosis coefficient $\Gamma$ is calculated by comparison to numerical simulations of the stochastic process, in such a way that the ensemble average of the total number of active tips equals $\int \rho(\mathbf{x}, t) d \mathbf{x}$. Anastomosis is a "killing process" that occurs naturally in two dimensional reductions of the model. In three dimensions, anastomosis becomes more complex, as we may have to associate diameters to blood vessels and introduce some proximity criterion for vessel fusion. This may change the time integral of $p(\mathbf{x}, \mathbf{v}, t)$ in Eq. (1) to a time integral of a convolution product of $p$ with some kernel that has a sharp peak inside a sphere of radius twice the tip radius. Ref. [10] contains a discussion of this point. We will set $N=2$ henceforth. Generation of new tips 
due to vessel tip branching is described by the source term $\alpha(C) \delta_{\sigma_{v}}\left(\mathbf{v}-\mathbf{v}^{0}\right) p$. This ignores secondary branching from an existing blood vessel. In principle, $\delta_{\sigma_{v}}\left(\mathbf{v}-\mathbf{v}^{0}\right)$ should be a Dirac measure supported at a point $\mathbf{v}^{0}$, where $\mathbf{v}^{0}$ is a typical sprouting velocity for the tips. Existence of solutions, as well as regularity, uniqueness and stability results, have been established in unbounded and bounded domains $[13,14]$ when the delta function is approximated by a smooth, positive, integrable and bounded function. Here, we take $\delta_{\sigma_{v}}\left(\mathbf{v}-\mathbf{v}^{0}\right)$ to be a Gaussian centered about $\mathbf{v}^{0}$ :

$$
\delta_{\sigma_{v}}\left(\mathbf{v}-\mathbf{v}^{0}\right)=\frac{1}{\pi \sigma_{v}^{2}} e^{-\left|\mathbf{v}-\mathbf{v}^{0}\right|^{2} / \sigma_{v}^{2}} .
$$

\begin{tabular}{cccccccccc}
\hline$\delta_{1}$ & $\beta$ & $A$ & $\Gamma$ & $\Gamma_{1}, q_{1}$ & $\kappa$ & $\chi$ & $\eta$ & $\epsilon$ & $\sigma_{v}$ \\
0.255 & 5.88 & 22.42 & 0.135 & 1 & 0.0045 & 0.002 & 15 & 0.001 & 0.08 \\
\hline
\end{tabular}

Table 1: Dimensionless parameters.

Capasso and Morale first introduced counting densities of actively moving vessel tips instead of tracking densities of vessel endothelial cells [8]. However, they could not properly account for branching and not at all for anastomosis in the deterministic description. The source terms in (1) were first derived in [3]. Previous authors did not incorporate them to equations for probability densities (Fokker-Planck equations) that did not take into account generation of new tips and their annihilation due to anastomosis; see the review papers [27, 35]. The generation and annihilation source terms are crucial to explain soliton-like behavior of solutions to the system of equations (1)-(3) and of ensemble averages of the associated stochastic process $[4,5,6]$. These references consider fluxes of the form $|\mathbf{j}|$ where

$$
\mathbf{j}(\mathbf{x}, t)=\int_{\mathbb{R}^{N}} \mathbf{v} p(\mathbf{x}, \mathbf{v}, t) d \mathbf{v},
$$

and $|\cdot|$ denotes the euclidean norm. As pointed out in [13], working with $|\mathbf{j}|$ may bring about uniqueness problems for some data since the nonlinearity is not Lipschitz. Defining $j(\mathbf{x}, t)=\int_{\mathbb{R}^{N}}|\mathbf{v}| p(\mathbf{x}, \mathbf{v}, t) d \mathbf{v}$ instead, this technical problem disappears. For this flux $j$ and general integrable initial densities, reference [13] establishes existence and uniqueness locally in time. Global in time existence $[13,14]$ is only proved when a velocity cut-off in terms of the Fermi-Dirac distribution is incorporated in the definition of $j$, as we do here in equation (5). The cut-off enforces the fact that cell velocities are limited and small. The constant $\eta$ is large, typically $\eta>10$, while $\epsilon \ll 1$. The FokkerPlanck operator represents blood vessel extension. The chemotactic force $\mathbf{F}(C)$ depends on the average tip speed through $j$ because consumption of tumor angiogenic factor is mostly performed by the additional endothelial cells that promote vessel extensions [3, 8, 42]. Other mechanisms of cellular motion such as haptotaxis can be included in $\mathbf{F}(C)$ as indicated in $[6,8]$. 
Let us fix a geometry for our analysis and simulations. We consider a slab $(0,1) \times \mathbb{R}$, and set $\mathbf{x}=\left(x_{1}, x_{2}\right), \mathbf{v}=\left(v_{1}, v_{2}\right)$. On the walls $x_{1}=0$ and $x_{1}=1$, we impose Neumann boundary conditions for $C$ :

$$
\frac{\partial}{\partial \mathbf{n}} C\left(0, x_{2}, t\right)=0, \quad \frac{\partial}{\partial \mathbf{n}} C\left(1, x_{2}, t\right)=c_{L}(t) e^{-a^{2} x_{2}^{2}}, \quad t>0, x_{2} \in \mathbb{R},
$$

where $c_{L}(t)>0$ to represent the influx of tumor angiogenic factor produced by the core of the tumor and $1 / a$ is a characteristic length thereof. This function decreases with time as new blood vessels reach the tumor. Moreover, $C \rightarrow 0$ as $\left|x_{2}\right| \rightarrow \infty$. The following nonlocal boundary conditions hold for $p$ :

$$
\begin{aligned}
& p^{+}\left(0, x_{2}, v_{1}, v_{2}, t\right)=\frac{e^{-\left|\mathbf{v}-\mathbf{v}^{0}\right|^{2}}}{\int_{0}^{\infty} \int_{-\infty}^{+\infty} v_{1}^{\prime} e^{-\left|\mathbf{v}^{\prime}-\mathbf{v}^{0}\right|^{2}} d v_{1}^{\prime} d v_{2}^{\prime}}\left[j_{0}\left(x_{2}, t\right)\right. \\
& \left.-\int_{-\infty}^{0} \int_{-\infty}^{+\infty} v_{1}^{\prime} p^{-}\left(0, x_{2}, v_{1}^{\prime}, v_{2}^{\prime}, t\right) d v_{1}^{\prime} d v_{2}^{\prime}\right]=S_{0}(p), \quad t, v_{1}>0, x_{2}, v_{2} \in \mathbb{R} \\
& p^{-}\left(1, x_{2}, v_{1}, v_{2}, t\right)=\frac{e^{-\left|\mathbf{v}-\mathbf{v}^{0}\right|^{2}}}{\int_{-\infty}^{0} \int_{-\infty}^{+\infty} e^{-\left|\mathbf{v}^{\prime}-\mathbf{v}^{0}\right|^{2}} d v_{1}^{\prime} d v_{2}^{\prime}}\left[\rho_{L}\left(x_{2}, t\right)\right. \\
& \left.-\int_{0}^{+\infty} \int_{-\infty}^{+\infty} p^{+}\left(1, x_{2}, v_{1}^{\prime}, v_{2}^{\prime}, t\right) d v_{1}^{\prime} d v_{2}^{\prime}\right]=S_{L}(p), \quad t>0, v_{1}<0, x_{2}, v_{2} \in \mathbb{R} .
\end{aligned}
$$

Here, $p^{+}$denotes the values of $p$ for positive $v_{1}$ and $p^{-}$the values of $p$ for negative $v_{1}$. For a fixed velocity $\mathbf{v}^{0}=\left(v_{1}^{0}, v_{2}^{0}\right)$

$$
\begin{array}{r}
j_{0}\left(x_{2}, t\right)=v_{1}^{0} \alpha\left(C\left(0, x_{2}, t\right)\right) p\left(0, x_{2}, v_{1}^{0}, v_{2}^{0}, t\right), \quad v_{1}^{0} \geq 0, \\
\rho_{L}\left(x_{2}, t\right)=\rho\left(1, x_{2}, t\right)=\int_{-\infty}^{+\infty} \int_{-\infty}^{+\infty} p\left(1, x_{2}, v_{1}^{\prime}, v_{2}^{\prime}, t\right) d v_{1}^{\prime} d v_{2}^{\prime} .
\end{array}
$$

These boundary conditions differ from those employed in slab geometries in $[4,5,6]$ because we must take into account the presence of velocity cut-offs in the flux (5). Conditions with a similar structure were used in [14] for theoretical studies in annular domains. The operators $S_{0}$ and $S_{L}$ take positive values for positive $p$ and $C$. Notice that $j_{0} \geq 0$ and the second integral in (9) involves only negative values of $v_{1}^{\prime}$ multiplied by a minus sign. Subtracting the two integrals appearing in (10) we obtain $\int_{-\infty}^{0} \int_{-\infty}^{+\infty} p^{+}\left(1, x_{2}, v_{1}^{\prime}, v_{2}^{\prime}, t\right) d v_{1}^{\prime} d v_{2}^{\prime}$ due to (12).

The structure of the boundary conditions for $p$ is consistent with the general theory of transport operators. Diffusion being absent in the $\mathbf{x}$ variable, we need boundary conditions of the form:

$$
p^{-}(\mathbf{x}, \mathbf{v}, t)=g(\mathbf{x}, \mathbf{v}, t) \quad \text { on } \Sigma_{T}^{-},
$$

where $\Sigma_{T}^{ \pm}=(0, T) \times \Gamma^{ \pm}$, with $\Gamma^{ \pm}=\{(\mathbf{x}, \mathbf{v}) \in \partial \Omega \times \mathbb{R} \mid \pm \mathbf{v} \cdot \hat{\mathbf{n}}(\mathbf{x})>0\}$, and $\hat{\mathbf{n}}(\mathbf{x})$ stands for the unit normal onto the boundary $\partial \Omega=\left\{x_{1}=0\right\} \cup\left\{x_{1}=1\right\}$. We denote by $p^{+}$and $p^{-}$the traces of $p$ on $\Sigma_{T}^{+}$and $\Sigma_{T}^{-}$, respectively. In our geometry, the boundary conditions for $p$ are defined using the magnitudes that 
can actually be measured: the marginal tip density $\rho=\int p d \mathbf{v}$ in the inner boundary and the flux of blood vessels $\hat{\mathbf{n}} \cdot \mathbf{J}=\int \hat{\mathbf{n}} \cdot \mathbf{v} p d \mathbf{v}$ in the outer boundary.

Equation (1) evokes Vlasov-Poisson-Fokker-Planck (VPFP) systems, with two remarkable differences. First, the chemotactic force field $\mathbf{F}(C)$ is not obtained solving a Poisson equation with source $\rho(p)$. It depends on the gradient of solutions $C$ of heat equations involving the modified average tip speed $j(p)$. Second, competing sources are present: the nonlocal in time integrodifferential anastomosis sink $-p \int_{0}^{t} \rho$ competes with the creation source $\alpha(C) \delta_{\sigma_{v}} p$. Unless handled properly, the presence of the sink may interfere with the expected positive sign of the density $p$.

Our goal here is to devise a robust first order explicit scheme for the nonlinear problem and boundary conditions under consideration. Available well posedness theories for this type of problems $[13,14]$ rely on iterative procedures to establish existence of solutions. These procedures freeze the nonlocal coefficients to guarantee that we are dealing with positive linear operators and that a maximum principle holds for $p$ and $C$. The resulting bounds allow us to employ compactness arguments to deduce the existence of limiting solutions. To devise numerical schemes, one might prove convergence of the whole sequence of iterates $p_{n}, C_{n}$ in some norm (which is hard in bounded domains) and resort to numerical schemes for linear problems to approximate $p_{n}$ and $C_{n}$ in $[0, T]$ at each iteration. Even if convergence as $n$ grows was theoretically achieved, this procedure is likely to be inefficient from the computational point of view.

Many schemes have been proposed for other kinetic problems, exploiting different features of the specific equations [19]. An overview of numerical methods for Vlasov-Poisson (VP) and Vlasov-Maxwell (VM) systems is given in [20]: particle in cell methods [17, 43], semi-lagrangian approaches [38] generalizing splitting methods [16], WENO interpolation [33], discontinuous Galerkin techniques [36], conservative flux based methods [21], energy conserving finite differences [22]. VP and VM models incorporate a transport operator coupled to either Poisson or Maxwell problems. However, they lack the degenerate diffusion term present in Fokker-Planck (FP) type models. Numerical schemes for FokkerPlanck equations in several dimensions are proposed for instance in [37, 15]; they often exploit finite difference approaches [29]. Variants of Fokker-Planck operators are tackled in $[7,41]$.

Finite difference and finite element methods for Vlasov-Fokker-Planck models are studied in [1, 2, 28]. As said before, VPFP models resemble our system, with some significant differences in the coupling and the nonlocal terms. For higher dimensional VPFP systems, random particle methods [25], deterministic particle methods combined with splitting of diffusion and convection operators [26], as well as finite differences combined with a change of variables [44] have been proposed. These schemes are often tested by checking convergence to a stationary solution, and tend to enforce periodic boundary conditions for simplicity. In our case, the model behavior we need to track is a traveling soliton, whose existence is ensured by the competition of the anastomosis and creation terms, driven by the coupling with the concentration of tumor angiogenic factor [4]. This soliton-like wave for the density of tips emerges at a pre-existing blood 
vessel (after a formation stage) and moves until it reaches the tumor. We will see that, in the slab geometry we consider here, finite difference approaches provide a robust choice to preserve positivity and handle the coupling with the diffusion equation as well as the nonlocal coefficients. Notice that the anastomosis sink is nonlocal in time, an unusual feature in the above mentioned kinetic models.

The paper is organized as follows. In Section 2 we describe the numerical scheme, proving stability and convergence results. The scheme has order at most one and enjoys some positivity properties. Sections 3 and 4 check the performance of the scheme by comparing with explicit soliton-like solutions constructed by asymptotic methods and with direct solutions of stochastic equations. Unlike $[4,5,6]$, we consider here fluxes $(5)$ in which the euclidean norm affects the velocity $|\mathbf{v}|$ instead of a vector flux $|\mathbf{j}|$ as in (7), and, additionally, a velocity cut-off is present. This avoids uniqueness issues for some classes of

initial data due to lack of lipschitzianity of the flux and ensures existence of global stable solutions for the kinetic model. In the whole space, uniqueness of such weak solutions has been proved. We also investigate the influence of velocity cut-offs in the profiles. Available global in time existence proofs for the kinetic model, as well as our convergence proofs for the numerical scheme, require velocity cut-offs in the flux $j$. For initial data with exponential velocity decay, numerical simulations provide consistent solutions in absence of such cutoffs. The numerical schemes perform well in both cases and the solutions show little dependence on the presence of the cut-offs. This suggests that the kinetic model should have stable global in time solutions for data decaying exponentially fast even if we do not introduce velocity cut-offs in the fluxes. This is in agreement with the experimental observation that the velocities of the vessel tips remain in a certain velocity range, reasonable for cells. Section 5 comments on conclusions and perspectives. Among open problems, we may list developing efficient higher order schemes, and exploring whether ideas implemented to simulate other kinetic models adapt to the angiogenesis framework or encounter structural limitations. Monotonicity properties may be a basis for the development of higher order schemes [31]. Lastly, Appendix A contains the derivation of soliton formulas for the flux $j$ used in this paper.

\section{Stability and convergence properties of the numerical scheme}

A robust finite difference scheme for system (1)-(5) follows by choosing upwind type discretizations for space and velocity derivatives in the transport operators, which take into account the propagation sign. The time integral is replaced by Riemann sums. The velocity integrals use two-dimensional Simpson rules. Time derivatives are discretized using progressive finite differences, leading to an explicit scheme.

We set as spatial domain $\Omega_{\mathbf{x}}=[0, X] \times[-\tau X, \tau X], \tau, X>0$, velocity domain $\Omega_{\mathbf{v}}=[-V, V] \times[-V, V], V>0$, and temporal interval $[0, T], T>0$. To simplify, we choose the same steps $\delta x$ and $\delta v$ for the two spatial and velocity components, 
$\delta t$ stands for the time step. We mesh the computational domain using the grid:

$$
\begin{aligned}
& x_{1, n_{1}}=x_{1,0}+n_{1} \delta x, \quad x_{2, n_{2}}=x_{2,0}+n_{2} \delta x, \quad n_{i}=0, \ldots, N_{i}, \quad i=1,2, \\
& v_{1, m_{1}}=v_{1,0}+m_{1} \delta v, \quad v_{2, m_{2}}=v_{2,0}+m_{2} \delta v, \quad m_{i}=0, \ldots, M, \quad i=1,2 \text {, } \\
& t_{\ell}=\ell \delta t, \quad \ell=0, \ldots, \mathcal{L},
\end{aligned}
$$

and discretize using forward differences in time, standard second order finite differences for laplacians, and upwind discretization for first order derivatives in space and velocity of $p$ :

$$
\begin{aligned}
& \frac{\partial p}{\partial t}\left(t_{\ell}\right)=\frac{p\left(t_{\ell+1}\right)-p\left(t_{\ell}\right)}{\delta t}+O(\delta t), \\
& F_{i, \mathbf{n}}^{\ell} \frac{\partial p}{\partial v_{i}}\left(v_{i, m_{i}}\right)=\left\{\begin{array}{l}
F_{i, \mathbf{n}}^{\ell} \frac{p\left(v_{i, m_{i}+1}\right)-p\left(v_{i, m_{i}}\right)}{\delta v}+O(\delta v), \text { if } F_{i, \mathbf{n}}^{\ell} \leq 0, \\
F_{i, \mathbf{n}}^{\ell} \frac{p\left(v_{i, m_{i}}\right)-p\left(v_{i, m_{i}-1}\right)}{\delta v}+O(\delta v), \text { if } F_{i, \mathbf{n}}^{\ell}>0,
\end{array} \quad i=1,2,\right. \\
& v_{i, m_{i}} \frac{\partial p}{\partial v_{i}}\left(v_{i, m_{i}}\right)=\left\{\begin{array}{l}
v_{i, m_{i}} \frac{p\left(v_{i, m_{i}+1}\right)-p\left(v_{i, m_{i}}\right)}{\delta v}+O(\delta v), \text { if } v_{i, m_{i}} \leq 0, \\
v_{i, m_{i}} \frac{p\left(v_{i, m_{i}}\right)-p\left(v_{i, m_{i}-1}\right)}{\delta v}+O(\delta v), \text { if } v_{i, m_{i}}>0,
\end{array} \quad i=1,2,\right. \\
& \frac{\partial^{2} p}{\partial v_{i}^{2}}\left(v_{i, m_{i}}\right)=\frac{p\left(v_{i, m_{i}+1}\right)-2 p\left(v_{i, m_{i}}\right)+p\left(v_{i, m_{i}-1}\right)}{\delta v^{2}}+O\left(\delta v^{2}\right), i=1,2, \\
& v_{i, m_{i}} \frac{\partial p}{\partial x_{i}}\left(x_{i, n_{i}}\right)=\left\{\begin{array}{l}
v_{i, m_{i}} \frac{p\left(x_{i, n_{i}+1}\right)-p\left(x_{i, n_{i}}\right)}{\delta x}+O(\delta x), \text { if } v_{i, m_{i}} \leq 0, \\
v_{i, m_{i}} \frac{p\left(x_{i, n_{i}}\right)-p\left(x_{i, n_{i}-1}\right)}{\delta x}+O(\delta x), \text { if } v_{i, m_{i}}>0,
\end{array} \quad i=1,2,\right. \\
& \frac{\partial C}{\partial t}\left(t_{\ell}\right)=\frac{C\left(t_{\ell+1}\right)-C\left(t_{\ell}\right)}{\delta t}+O(\delta t), \\
& \frac{\partial^{2} C}{\partial x_{i}^{2}}\left(x_{i, n_{i}}\right)=\frac{C\left(x_{i, n_{i}+1}\right)-2 C\left(x_{i, n_{i}}\right)+C\left(x_{i, n_{i}-1}\right)}{\delta x^{2}}+O\left(\delta x^{2}\right), i=1,2,
\end{aligned}
$$

the coefficients $F_{i, \mathbf{n}}^{\ell}=F_{i}\left(x_{1, n_{1}}, x_{2, n_{2}}, t_{\ell}\right), \mathbf{n}=\left(n_{1}, n_{2}\right)$, being force terms. We set $\mathbf{x}_{\mathbf{n}}=\left(x_{1, n_{1}}, x_{2, n_{2}}\right)$ and $\mathbf{v}_{\mathbf{m}}=\left(v_{1, m_{1}}, v_{2, m_{2}}\right)$. The derivatives $\frac{\partial\left(v_{i} p\right)}{\partial v_{i}}$ are treated like $v_{i} \frac{\partial p}{\partial v_{i}}+p$. The time integral becomes

$$
\int_{0}^{t} \rho(p)(s) d s=\delta t \sum_{\ell=0}^{\mathcal{L}-1} \rho\left(p\left(t_{\ell}\right)\right)+O\left(\delta t^{2}\right) .
$$

Using the composite Simpson rule in two dimensions, the velocity integrals for $q=p, w(\mathbf{v}) p$, with weight $w(\mathbf{v})=\frac{|\mathbf{v}|}{1+e^{\left(\left|\mathbf{v}-\mathbf{v}^{0}\right|^{2}-\eta\right) / \varepsilon}}$ fixed in (5), take the form:

$$
\int_{\mathbb{R}^{N}} q(\mathbf{v}) d \mathbf{v} \sim \int_{\Omega_{\mathbf{v}}} q(\mathbf{v}) d \mathbf{v}=\delta v^{2} \sum_{m_{1}, m_{2}=0}^{M} a_{m_{1}, m_{2}} q\left(v_{1, m_{1}}, v_{2, m_{2}}\right)+O\left(\delta v^{4}\right)
$$

where $0 \leq a_{m_{1}, m_{2}} \leq \tilde{a}=\frac{16}{9}$. The nonlocal boundary conditions (9)-(10) are also discretized using the composite Simpson rule, while the Neumann boundary conditions (8) are discretized by means of first order forward and backward finite differences at $x_{1}=0$ and $x_{1}=1$, respectively. 
We introduce the above approximations in system (1)-(5), neglect the error terms and consider the resulting system of difference equations. The truncature error when discretizing the differential operators is of order $O(\delta t+\delta x+\delta v)$ for $p$ and $O\left(\delta t+\delta x^{2}\right)$ for $C$. The Neumann boundary conditions lower the order to $O(\delta t+\delta x)$ for $C$. The discretization of the integral operators provide additional terms of orders $\delta t^{2}$ and $\delta v^{4}$.

Notice that we have chosen an explicit scheme in time, while implicit schemes are usually expected to enjoy better stability properties. The explicit scheme has monotonicity properties that ensure the positivity of both, densities $p_{\mathbf{n}, \mathbf{m}}^{\ell}$ and concentrations $C_{\mathbf{n}}^{\ell}$, under simple hypotheses. Instead of this, due the presence of nonlocal terms in the equations and in the boundary conditions, an implicit scheme would require solving linear systems involving about $N_{1} \times N_{2} \times M^{2}+N_{1} \times$ $N_{2}$ unknowns, with full blocks in the matrices due to the coupling in velocity. This increases the cost per iteration and makes it hard to establish positivity properties. Without positive densities, and depending how we discretize the term that is nonlocal in time, even stability may not be an obvious property of the implicit scheme. However, once positivity of the densities are ensured, one might try different schemes for the concentrations.

We will study next stability and convergence conditions for our explicit scheme under additional assumptions motivated by the structure of the physically relevant solutions studied in Section 3. Since the initial data decay exponentially fast with respect to the velocity, we impose zero boundary conditions for the density on $\partial \Omega_{\mathbf{v}}$. The density essentially vanishes on $\partial \Omega_{\mathbf{x}}$ during the migration of the soliton from the vessel to the tumor too (see Figure 2 (b) for $\rho$ ), except, perhaps at some early or late stages of the simulation, on the wall close to the vessel, that is, $x_{1}=0$ and on the wall containing the tumor, i.e., $x_{1}=1$.

Let us first make the change of variables:

$$
p=e^{\lambda t} \tilde{p}, \quad C=e^{\nu t} \tilde{C},
$$

where $\lambda>0$ and $\nu \geq 0$ may be chosen large enough. Then $\tilde{p}$ and $\tilde{C}$ satisfy the equations

$$
\begin{aligned}
\frac{\partial}{\partial t} \tilde{p}= & {\left[-\lambda+\alpha\left(e^{\nu t} \tilde{C}\right) \delta_{\mathbf{v}^{0}}-\Gamma \int_{0}^{t} d s \int d \mathbf{v}^{\prime} e^{\lambda s} \tilde{p}\right] \tilde{p}-\mathbf{v} \cdot \nabla_{\mathbf{x}} \tilde{p} } \\
& +\beta \operatorname{div}_{\mathbf{v}}(\mathbf{v} \tilde{p})-\beta \operatorname{div}_{\mathbf{v}}\left[\mathbf{F}\left(e^{\nu t} \tilde{C}\right) \tilde{p}\right]+\frac{\beta}{2} \Delta_{\mathbf{v}} \tilde{p}, \\
\frac{\partial}{\partial t} \tilde{C}= & \kappa \Delta_{\mathbf{x}} \tilde{C}-\left[\nu+\chi \int w(\mathbf{v}) e^{\lambda t} \tilde{p} d \mathbf{v}\right] \tilde{C}, \\
\tilde{p}(0)= & p_{0}, \quad \tilde{C}(0)=c_{0},
\end{aligned}
$$

with data in the boundary conditions scaled by the exponential factors, that is, multiplied by $e^{-\nu t}$ and $e^{-\lambda t}$ respectively. Here and henceforth we have used $\delta_{\mathbf{v}^{0}}(\cdot)=\delta_{\sigma_{v}}\left(\cdot-\mathbf{v}^{0}\right)$. We drop the symbol ${ }^{\sim}$ in the sequel for ease of notation. 
We denote by $p_{\mathbf{n}, \mathbf{m}}^{\ell}=p_{n_{1}, n_{2}, m_{1}, m_{2}}^{\ell}$ and $C_{\mathbf{n}}^{\ell}=C_{n_{1}, n_{2}}^{\ell}$ the approximations of the continuous fields $p\left(x_{1, n_{1}}, x_{2, n_{2}}, v_{1, m_{1}}, v_{2, m_{2}}, t_{\ell}\right)$ and $C\left(x_{1, n_{1}}, x_{2, n_{2}}, t_{\ell}\right)$, respectively. Let us introduce the discrete operators:

$$
\begin{array}{r}
-v_{1, m_{1}} D_{m_{1}} p_{\mathbf{n}, \mathbf{m}}^{\ell}=v_{1, m_{1}}^{-} \frac{p_{\mathbf{n}, m_{1}, m_{2}}^{\ell}-p_{\mathbf{n}, m_{1}-1, m_{2}}^{\ell}}{\delta v}-v_{1, m_{1}}^{+} \frac{p_{\mathbf{n}, m_{1}+1, m_{2}}^{\ell}-p_{\mathbf{n}, m_{1}, m_{2}}^{\ell}}{\delta v}, \\
F_{1, \mathbf{n}}^{\ell} D_{m_{1}} p_{\mathbf{n}, \mathbf{m}}^{\ell}=F_{1, \mathbf{n}}^{\ell,+} \frac{p_{\mathbf{n}, m_{1}, m_{2}}^{\ell}-p_{\mathbf{n}, m_{1}-1, m_{2}}^{\ell}}{\delta v}-F_{1, \mathbf{n}}^{\ell,-} \frac{p_{\mathbf{n}, m_{1}+1, m_{2}}^{\ell}-p_{\mathbf{n}, m_{1}, m_{2}}^{\ell}}{\delta v}, \\
v_{1, m_{1}} D_{n_{1}} p_{\mathbf{n}, \mathbf{m}}^{\ell}=v_{1, m_{1}}^{+} \frac{p_{n_{1}, n_{2}, \mathbf{m}}^{\ell}-p_{n_{1}-1, n_{2}, \mathbf{m}}^{\ell}}{\delta x}-v_{1, m_{1}}^{-} \frac{p_{n_{1}+1, n_{2}, \mathbf{m}}^{\ell}-p_{n_{1}, n_{2}, \mathbf{m}}^{\ell}}{\delta x}, \\
D_{n_{1}}^{2} C_{\mathbf{n}}^{\ell}=\frac{C_{n_{1}+1, n_{2}}^{\ell}-2 C_{n_{1}, n_{2}}^{\ell}+C_{n_{1}-1, m_{2}}^{\ell}}{\delta x^{2}},
\end{array}
$$

where $\mathbf{n}=\left(n_{1}, n_{2}\right)$ and $\mathbf{m}=\left(m_{1}, m_{2}\right)$. The symbols ${ }^{+}$and ${ }^{-}$denote here positive and negative parts, respectively. Analogous definitions apply to differences with respect to $m_{2}$ and $n_{2}$. The scheme can then be written as follows:

$$
\begin{aligned}
& \frac{p_{\mathbf{n}, \mathbf{m}}^{\ell+1}-p_{\mathbf{n}, \mathbf{m}}^{\ell}}{\delta t}+\sum_{i=1}^{2}\left[v_{i, m_{i}} D_{n_{i}} p_{\mathbf{n}, \mathbf{m}}^{\ell}+\beta\left(F_{i, \mathbf{n}}^{\ell}-v_{i, m_{i}}\right) D_{m_{i}} p_{\mathbf{n}, \mathbf{m}}^{\ell}-\frac{\beta}{2} D_{m_{i}}^{2} p_{\mathbf{n}, \mathbf{m}}^{\ell}\right] \\
= & {\left[-\lambda+2 \beta+\alpha\left(e^{\nu t_{\ell}} C_{\mathbf{n}}^{\ell}\right) \delta_{\mathbf{v}^{0}}\left(v_{\mathbf{m}}\right)-\Gamma \delta t \delta v^{2} \sum_{k=0}^{\ell} \sum_{m_{1}^{\prime}, m_{2}^{\prime}=0}^{M} e^{\lambda t_{k}} a_{\mathbf{m}^{\prime}} p_{\mathbf{n}, \mathbf{m}^{\prime}}^{k}\right] p_{\mathbf{n}, \mathbf{m}}^{\ell} } \\
& \frac{C_{\mathbf{n}}^{\ell+1}-C_{\mathbf{n}}^{\ell}}{\delta t}-\kappa \sum_{i=1}^{2} D_{n_{i}}^{2} C_{\mathbf{n}}^{\ell}=-\left[\nu+\chi \delta v^{2} \sum_{m_{1}^{\prime}, m_{2}^{\prime}=0}^{M} e^{\lambda t_{\ell}} a_{\mathbf{m}^{\prime}} w\left(v_{\mathbf{m}^{\prime}}\right) p_{\mathbf{n}, \mathbf{m}^{\prime}}^{\ell}\right] C_{\mathbf{n}}^{\ell},
\end{aligned}
$$

where $F_{i, \mathbf{n}}^{\ell}=F_{i}\left(x_{1, n_{1}}, x_{2, n_{2}}, t_{\ell}\right)$. These are explicit schemes yielding expressions for the values of the unknowns in a new time level provided the values in the previous ones are known:

$$
\begin{aligned}
& p_{\mathbf{n}, \mathbf{m}}^{\ell+1}=\left(1-\delta t b_{\mathbf{n}, \mathbf{m}}^{\ell}-2 \beta \frac{\delta t}{\delta v^{2}}-\left(v_{1, m_{1}}^{-}+v_{1, m_{1}}^{+}+v_{2, m_{2}}^{-}+v_{2, m_{2}}^{+}\right) \frac{\delta t}{\delta x}\right) p_{\mathbf{n}, \mathbf{m}}^{\ell} \\
&+\frac{\delta t}{\delta v^{2}} \frac{\beta}{2}\left(p_{\mathbf{n}, m_{1}+1, m_{2}}^{\ell}+p_{\mathbf{n}, m_{1}-1, m_{2}}^{\ell}+p_{\mathbf{n}, m_{1}, m_{2}+1}^{\ell}+p_{\mathbf{n}, m_{1}, m_{2}-1}^{\ell}\right)+ \\
& \frac{\delta t}{\delta v} \beta\left(F_{1, \mathbf{n}}^{\ell,-}\left(p_{\mathbf{n}, m_{1}+1, m_{2}}^{\ell}-p_{\mathbf{n}, m_{1}, m_{2}}^{\ell}\right)+F_{1, \mathbf{n}}^{\ell,+}\left(-p_{\mathbf{n}, m_{1}, m_{2}}^{\ell}+p_{\mathbf{n}, m_{1}-1, m_{2}}^{\ell}\right)\right)+ \\
& \frac{\delta t}{\delta v} \beta\left(F_{2, \mathbf{n}}^{\ell,-}\left(p_{\mathbf{n}, m_{1}, m_{2}+1}^{\ell}-p_{\mathbf{n}, m_{1}, m_{2}}^{\ell}\right)+F_{2, \mathbf{n}}^{\ell,+}\left(-p_{\mathbf{n}, m_{1}, m_{2}}^{\ell}+p_{\mathbf{n}, m_{1}, m_{2}-1}^{\ell}\right)\right)+ \\
& \frac{\delta t}{\delta v} \beta\left(v_{1, m_{1}}^{+}\left(p_{\mathbf{n}, m_{1}+1, m_{2}}^{\ell}-p_{\mathbf{n}, m_{1}, m_{2}}^{\ell}\right)+v_{1, m_{1}}^{-}\left(-p_{\mathbf{n}, m_{1}, m_{2}}^{\ell}+p_{\mathbf{n}, m_{1}-1, m_{2}}^{\ell}\right)\right)+ \\
& \frac{\delta t}{\delta v} \beta\left(v_{2, m_{2}}^{+}\left(p_{\mathbf{n}, m_{1}, m_{2}+1}^{\ell}-p_{\mathbf{n}, m_{1}, m_{2}}^{\ell}\right)+v_{2, m_{2}}^{-}\left(-p_{\mathbf{n}, m_{1}, m_{2}}^{\ell}+p_{\mathbf{n}, m_{1}, m_{2}-1}^{\ell}\right)\right)+ \\
& \frac{\delta t}{\delta x}\left(v_{1, m_{1}}^{-} p_{n_{1}+1, n_{2}, \mathbf{m}}^{\ell}+v_{1, m_{1}}^{+} p_{n_{1}-1, n_{2}, \mathbf{m}}^{\ell}+v_{2, m_{2}}^{-} p_{n_{1}, n_{2}+1, \mathbf{m}}^{\ell}+v_{2, m_{2}}^{+} p_{n_{1}, n_{2}-1, \mathbf{m}}^{\ell}\right),
\end{aligned}
$$




$$
\begin{array}{r}
C_{\mathbf{n}}^{\ell+1}=\left(1-\delta t e_{\mathbf{n}}^{\ell}-4 \frac{\delta t}{\delta x^{2}} \kappa\right) C_{\mathbf{n}}^{\ell}+ \\
\frac{\delta t}{\delta x^{2}} \kappa\left(C_{n_{1}+1, n_{2}}^{\ell}+C_{n_{1}-1, n_{2}}^{\ell}+C_{n_{1}, n_{2}+1}^{\ell}+C_{n_{1}, n_{2}-1}^{\ell}\right),
\end{array}
$$

where we have set

$$
\begin{array}{r}
b_{\mathbf{n}, \mathbf{m}}^{\ell}=\lambda-2 \beta-\alpha\left(e^{\nu t_{\ell}} C_{\mathbf{n}}^{\ell}\right) \delta_{\mathbf{v}^{0}}\left(v_{\mathbf{m}}\right)+\Gamma \delta t \delta v^{2} \sum_{k=0}^{\ell} \sum_{m_{1}^{\prime}, m_{2}^{\prime}=0}^{M} e^{\lambda t_{k}} a_{\mathbf{m}^{\prime}} p_{\mathbf{n}, \mathbf{m}^{\prime}}^{k}, \\
e_{\mathbf{n}}^{\ell}=\nu+\chi \delta v^{2} \sum_{m_{1}^{\prime}, m_{2}^{\prime}=0}^{M} e^{\lambda t \ell} a_{\mathbf{m}^{\prime}} w\left(v_{\mathbf{m}^{\prime}}\right) p_{\mathbf{n}, \mathbf{m}^{\prime}}^{\ell}
\end{array}
$$

We denote $j^{d}\left(p_{\mathbf{n}, \mathbf{m}}^{\ell}\right)=\delta v^{2} \sum_{m_{1}^{\prime}, m_{2}^{\prime}=0}^{M} e^{\lambda t_{\ell}} a_{\mathbf{m}^{\prime}} w\left(v_{\mathbf{m}^{\prime}}\right) p_{\mathbf{n}, \mathbf{m}^{\prime}}^{\ell}$. Here, the field $\mathbf{F}$ depends on $C$ in the following way:

$$
\mathbf{F}_{\mathbf{n}}^{\ell}=\mathbf{F}\left(e^{\nu t_{\ell}} C_{\mathbf{n}}^{\ell}\right)=h\left(e^{\nu t_{\ell}} C_{\mathbf{n}}^{\ell}\right) e^{\nu t_{\ell}}\left(\frac{C_{n_{1}+1, n_{2}}^{\ell}-C_{n_{1}, n_{2}}^{\ell}}{\delta x}, \frac{C_{n_{1}, n_{2}+1}^{\ell}-C_{n_{1}, n_{2}}^{\ell}}{\delta x}\right),
$$

where $h(C)=\frac{\delta_{1}}{\left(1+\Gamma_{1} C\right)^{q_{1}}}$ is defined in (4).

Finally, the nonlocal boundary conditions (9)-(10) become:

$$
\begin{aligned}
& p_{0, n_{2}, \mathbf{m}}^{\ell+1}=\frac{2}{\sqrt{\pi}} e^{-\left|\mathbf{v}_{\mathbf{m}}-\mathbf{v}^{0}\right|^{2}}\left[v_{1}^{0} \alpha\left(C_{0, n_{2}}^{\ell}\right) p_{0, n_{2}, m_{1}^{0}, m_{2}^{0}}^{\ell}\right. \\
& \left.-\delta v^{2} \sum_{m_{1}^{\prime}=0}^{M_{0}} \sum_{m_{2}^{\prime}=0}^{M} a_{\mathbf{m}^{\prime}} v_{1, m_{1}^{\prime}} p_{0, n_{2}, \mathbf{m}^{\prime}}^{\ell}\right]=S_{0}^{d}\left(p^{\ell}\right), \quad v_{1, m_{1}}>0, \\
& p_{N_{1}, n_{2}, \mathbf{m}}^{\ell+1}=\frac{2}{\pi} e^{-\left|\mathbf{v}_{\mathbf{m}}-\mathbf{v}^{0}\right|^{2}} \delta v^{2} \sum_{m_{1}^{\prime}=0}^{M_{0}} \sum_{m_{2}^{\prime}=0}^{M} a_{\mathbf{m}^{\prime}} p_{N_{1}, n_{2}, \mathbf{m}^{\prime}}^{\ell}=S_{L}^{d}\left(p^{\ell}\right), \quad v_{1, m_{1}}<0,
\end{aligned}
$$

where $M_{0}$ is such $v_{1, M_{0}} \leq 0$ and $v_{1, M_{0}+1}>0$ and $\left(m_{1}^{0}, m_{2}^{0}\right)$ denotes the indices for which $\left(v_{1, m_{1}^{0}}, v_{\left.2, m_{2}^{0}\right)}\right.$ is closest to $\left(v_{1}^{0}, v_{2}^{2}\right)=\mathbf{v}^{0}$. Notice that this fixed reference velocity is not necessarily a grid node, therefore the value of the density at it must be approximated. On the boundaries $n_{2}=0, m_{2}=M_{0}+1, \ldots, M$, and $n_{2}=N_{2}, m_{2}=0, \ldots, M_{0}$ we typically set $p_{\mathbf{n}, \mathbf{m}}^{\ell}=0$. On the artificial boundaries $m_{i}=0, M, i=1,2$, we set $p_{\mathbf{n}, \mathbf{m}}^{\ell}=0$. The Neumann boundary conditions (8) take the form:

$$
C_{0, n_{2}}^{\ell}-C_{1, n_{2}}^{\ell}=0, \quad C_{N_{1}, n_{2}}^{\ell}-C_{N_{1}-1, n_{2}}^{\ell}=\delta x c_{L}\left(t_{\ell}\right) e^{-a^{2}\left(x_{2, n_{2}}\right)^{2}} .
$$

On the boundaries $n_{2}=0, N_{2}$, we typically set $C_{\mathbf{n}}^{\ell}=0$.

We use these conditions for the numerical simulations in Section 3, though for the numerical analysis in this section we supplement (26) with known boundary data $g_{\mathbf{m}, \mathbf{n}}^{\ell}$ on the spatial boundaries. The whole set of equations can be written more compactly in matrix form. We relabel the unknowns at the interior points, 
setting $P_{\zeta}^{\ell}=p_{\mathbf{n}, \mathbf{m}}^{\ell}$, with $\zeta=n_{2}+\left(n_{1}-1\right)\left(N_{2}-1\right)+\left(m_{1}-1\right)\left(N_{1}-1\right)\left(N_{2}-1\right)+$ $\left(m_{2}-1\right)\left(N_{1}-1\right)\left(N_{2}-1\right)(M-1)$ and $C_{\xi}^{\ell}=C_{\mathbf{n}}^{\ell}$ with $\xi=n_{2}+\left(n_{1}-1\right)\left(N_{2}-1\right)$, which leads to the systems:

$$
\begin{array}{r}
P^{\ell+1}=\mathbf{A}_{1} P^{\ell}+\operatorname{diag}\left[\sum_{k=0}^{\ell} \mathbf{B}_{1}^{k} P^{k}+D_{1}^{\ell}\left(C^{\ell}\right)\right] P^{\ell}+\mathbf{D}_{2}^{\ell}\left(C^{\ell}\right) P^{\ell}+\tilde{Z}^{\ell}=\tilde{\mathbf{A}}_{1}^{\ell} P^{\ell}+\tilde{Z}^{\ell} \\
C^{\ell+1}=\mathbf{A}_{2} C^{\ell}+\operatorname{diag}\left[\mathbf{B}_{2}^{\ell} P^{\ell}\right] C^{\ell}+\tilde{Y}^{\ell}=\tilde{\mathbf{A}}_{2}^{\ell} C^{\ell}+\tilde{Y}^{\ell} .
\end{array}
$$

Here, we have set $\tilde{\mathbf{A}}_{1}^{\ell}=\mathbf{A}_{1}+\operatorname{diag}\left[\sum_{k=0}^{\ell} \mathbf{B}_{1}^{k} P^{k}+D_{1}^{\ell}\left(C^{\ell}\right)\right]+\mathbf{D}_{2}^{\ell}\left(C^{\ell}\right)$ and $\tilde{\mathbf{A}}_{2}^{\ell}=$ $\mathbf{A}_{2}+\operatorname{diag}\left[\mathbf{B}_{2}^{k} P^{\ell}\right]$, where diag stands for diagonal matrix. Comparing with the pointwise scheme (26)-(27), the matrices $\mathbf{A}_{1}$ and $\mathbf{A}_{2}$ represent the terms coming from the linear differential operators. The matrices $\mathbf{B}_{1}^{k}, k=0, \ldots, \ell$, and $\mathbf{B}_{2}^{\ell}$ represent the nonlocal contributions in expressions (28) for $b_{\mathbf{n}, \mathbf{m}}^{\ell}$ and $e_{\mathbf{n}}^{\ell}$, coming from the discretization of the anastomosis and flux integrals by Simpson rule. The vector $D_{1}^{\ell}\left(C^{\ell}\right)$ embodies the contribution of the nonlinear creation function $\alpha(C)$ to $b_{\mathbf{n}, \mathbf{m}}^{\ell}$ in (28). Finally, the matrix $\mathbf{D}_{2}\left(C^{\ell}\right)$ incorporates the contribution of the force field $\mathbf{F}(C)$. Notice that the negative matrices $\mathbf{B}_{1}^{k}, \mathbf{B}_{2}^{k}$ depend on $e^{\lambda t_{k}} \leq e^{\lambda T}$. The vectors $D_{1}^{\ell}\left(C^{\ell}\right)$ and matrices $\mathbf{D}_{2}^{\ell}\left(C^{\ell}\right)$ depend on $e^{\nu t_{k}} \leq e^{\nu T}$. The vectors $\tilde{Z}^{\ell}$ and $\tilde{Y}^{\ell}$ collect the contribution from the boundary data which enter the scheme (26)-(27) when boundary points are involved, fact that is also reflected in the structure of the matrices $\mathbf{A}_{1}, \mathbf{A}_{2}$ and $\mathbf{D}_{2}^{\ell}$. For instance, in (34) $A_{2}=\mathcal{I}+\kappa \frac{\delta t}{\delta x^{2}} \mathcal{N}$ where $\mathcal{I}=I_{\left(N_{1}-1\right) \times\left(N_{2}-1\right)}$ is the identity and $\mathcal{N}$ is a block tridiagonal matrix

$$
\mathcal{N}=\left(\begin{array}{ccccc}
\mathcal{T} & I & 0 & . & 0 \\
I & \mathcal{T} & I & . & 0 \\
. & . & . & . & . \\
0 & . & I & \mathcal{T} & I \\
0 & . & 0 & I & \mathcal{T}
\end{array}\right), \quad \text { with } \quad \mathcal{T}=\left(\begin{array}{ccccc}
-3 & 1 & 0 & . & 0 \\
1 & -4 & 1 & . & 0 \\
. & . & . & . & . \\
0 & . & 1 & -4 & 1 \\
0 & . & 0 & 1 & -3
\end{array}\right)
$$

a block of size $\left(N_{1}-1\right)^{2}, I=I_{N_{1}-1}$ the identity matrix, and

$$
\begin{aligned}
C^{\ell} & =\left(C_{1,1}^{\ell}, \ldots, C_{N_{1}-1,1}^{\ell}, \ldots, C_{1, j}^{\ell}, \ldots, C_{N_{1}-1, j}^{\ell} \ldots, C_{1, N_{2}-1}^{\ell}, \ldots, C_{N_{1}-1, N_{2}-1}^{\ell}\right), \\
\tilde{Y}^{\ell} & =\kappa \frac{\delta t}{\delta x}\left(0, \ldots, d_{1}^{\ell}, \ldots, 0, \ldots, d_{j}^{\ell}, \ldots, 0, \ldots, d_{N_{2}-1}^{\ell}\right) .
\end{aligned}
$$

Whereas information on signs and some norms follows directly from the pointwise scheme (26)-(27), the matrix identity (33)-(34) will be useful to write compactly the convergence proof.

Let us first establish some positivity and boundedness results deriving from maximum principles. The following result is straightforward from the definition of the scheme using an induction argument.

Proposition 2.1. Let us assume that

(I1) the initial values satisfy: 
- Positivity: $p_{\mathbf{n}, \mathbf{m}}^{0} \geq 0, C_{\mathbf{n}}^{0} \geq 0$, for $n_{i}=0, \ldots, N_{i}, m_{i}=0, \ldots, M$, $i=1,2$.

(I2) the boundary values satisfy:

- $p_{\mathbf{n}, \mathbf{m}}^{\ell}=0$ for $m_{i}=0, M, i=1,2$, for all $\ell \geq 0$,

$-p_{\mathbf{n}, \mathbf{m}}^{\ell}=g_{\mathbf{n}, \mathbf{m}}^{\ell} \geq 0$ for $n_{i}=0, m_{i}=M_{0}+1, \ldots, M$, and for $n_{i}=N_{i}$, $m_{i}=0, \ldots, M_{0}, i=1,2, \ell \geq 0$, where $M_{0}$ is such that $v_{i, M_{0}} \leq 0$ and $v_{i, M_{0}+1}>0$.

- $C_{\mathbf{n}}^{\ell}=d_{\mathbf{n}}^{\ell} \geq 0$ when $n_{2}=0, N_{2}$, for all $\ell \geq 0$,

$-C_{0, n_{2}}^{\ell}-C_{1, n_{2}}^{\ell}=0$ and $C_{N_{1}, n_{2}}^{\ell}-C_{N_{1}-1, n_{2}}^{\ell}=\delta x d_{N_{1}, n_{2}}^{\ell} \geq 0, n_{2}=$ $0, \ldots, N_{2}$, for all $\ell \geq 0$.

Moreover, the Dirichlet and Neumann boundary data $d_{\mathbf{n}}^{j}$ are generated from a $C^{2}\left([0, T] ; C^{4}\left(\bar{\Omega}_{\mathbf{x}}\right)\right)$ function.

If the following conditions hold

(HO) $\lambda \geq 2 \beta+\max \left|\alpha \delta_{\mathbf{v}^{0}}\right|$,

(H1) $B_{\mathbf{n}, \mathbf{m}}^{\ell}=1-\delta t b_{\mathbf{n}, \mathbf{m}}^{\ell}-2 \beta \frac{\delta t}{\delta v^{2}}-\left(v_{1, m_{1}}^{-}+v_{1, m_{1}}^{+}+v_{2, m_{2}}^{-}+v_{2, m_{2}}^{+}\right) \frac{\delta t}{\delta x}$

$-\left[\left(F_{1, \mathbf{n}}^{\ell,-}+F_{1, \mathbf{n}}^{\ell,+}+F_{2, \mathbf{n}}^{\ell,-}+F_{2, \mathbf{n}}^{\ell,+}\right)+\left(v_{1, m_{1}}^{-}+v_{1, m_{1}}^{+}+v_{2, m_{2}}^{-}+v_{2, m_{2}}^{+}\right)\right] \beta \frac{\delta t}{\delta v} \geq 0$,

(H2) $E_{\mathbf{n}}^{\ell}=1-\delta t e_{\mathbf{n}}^{\ell}-4 \frac{\delta t}{\delta x^{2}} \kappa \geq 0$,

(H3) $b_{\mathbf{n}, \mathbf{m}}^{\ell}, e_{\mathbf{n}}^{\ell} \geq 0$,

for $0 \leq \ell \leq \mathcal{L}$, then the scheme (26)-(27) satisfies the following properties:

- Positivity preservation: $p_{\mathbf{n}, \mathbf{m}}^{\ell} \geq 0, C_{\mathbf{n}}^{\ell} \geq 0$, for $0 \leq \ell \leq \mathcal{L}, n_{i}=0, \ldots, N_{i}$, $m_{i}=0, \ldots, M, i=1,2$. Moreover, if $p_{\mathbf{n}, \mathbf{m}}^{0}>0, C_{\mathbf{n}}^{0}>0, n_{i}=1, \ldots, N_{i}-1$, $m_{i}=1, \ldots, M-1, i=1,2$, then $p_{\mathbf{n}, \mathbf{m}}^{\ell}$ and $C_{\mathbf{n}}^{\ell}$ cannot vanish at such interior points for $0 \leq \ell \leq \mathcal{L}$.

- $\ell^{\infty}$ bound: for $0 \leq \ell \leq \mathcal{L}$

$$
\begin{aligned}
& \operatorname{Max}_{\mathbf{n}, \mathbf{m}}\left|p_{\mathbf{n}, \mathbf{m}}^{\ell}\right| \leq \operatorname{Max}_{\mathbf{n}, \mathbf{m}}\left|p_{\mathbf{n}, \mathbf{m}}^{0}\right|+\operatorname{Max}_{j \leq \ell-1} \operatorname{Max}_{\mathbf{n}, \mathbf{m}}\left|g_{\mathbf{n}, \mathbf{m}}^{j}\right|, \\
& \operatorname{Max}_{\mathbf{n}}\left|C_{\mathbf{n}}^{\ell}\right| \leq \operatorname{Max}_{\mathbf{n}}\left|C_{\mathbf{n}}^{0}\right|+f\left(d_{\mathbf{n}}^{j}, T\right),
\end{aligned}
$$

where $f\left(d_{\mathbf{n}}^{j}, T\right)$ is bounded independently of $\delta x$ and $\delta t$.

In particular, when $g_{\mathbf{n}, \mathbf{m}}^{\ell}=0$ and $d_{\mathbf{n}}^{\ell}=0$ for all $\mathbf{n}, \mathbf{m}, \ell$, we have:

- $\ell^{\infty}$ bound: $\left\|p_{\mathbf{n}, \mathbf{m}}^{\ell}\right\|_{\infty} \leq\left\|p_{\mathbf{n}, \mathbf{m}}^{0}\right\|_{\infty}$ and $\left\|C_{\mathbf{n}}^{\ell}\right\|_{\infty} \leq\left\|C_{\mathbf{n}}^{0}\right\|_{\infty}$ for $0 \leq \ell \leq \mathcal{L}$.

- Contractivity: $\left\|p_{\mathbf{n}, \mathbf{m}}^{\ell}\right\|_{\infty} \leq(1-(\lambda / 2) \delta t)\left\|p_{\mathbf{n}, \mathbf{m}}^{\ell-1}\right\|_{\infty}$, provided $\lambda / 2 \geq 2 \beta+$ $\max \left|\alpha \delta_{\mathbf{v}^{0}}\right|$ holds, and $\left\|C_{\mathbf{n}}^{\ell}\right\|_{\infty} \leq(1-\nu \delta t)\left\|C_{\mathbf{n}}^{\ell-1}\right\|_{\infty}$ when $\nu>0$, for $0 \leq$ $\ell \leq \mathcal{L}$ 
where the $\ell^{\infty}$ norms refer to spatial variables.

Furthermore, when the spatial boundary conditions for the densities are given by the discretized nonlocal operators (30)-(31) at $n_{1}=0, N_{1}$, and are set equal to zero at $n_{2}=0, N_{2}$, the sequences $p_{\mathbf{n}, \mathbf{m}}^{\ell}$ and $C_{\mathbf{n}}^{\ell}$ remain nonnegative for nonnegative data.

Proof. Let us assume that (H1), (H2) and (H3) hold for $\ell \geq 0$. Then, $p_{\mathbf{n}, \mathbf{m}}^{1} \geq 0$ and $C_{\mathbf{n}}^{1} \geq 0$ follow directly from (26)-(27), since the boundary conditions are nonnegative and (H1)-(H2) hold. By induction, we obtain $p_{\mathbf{n}, \mathbf{m}}^{\ell} \geq 0$ and $C_{\mathbf{n}}^{\ell} \geq 0$ for all $\ell \geq 0$. The fact that the coefficients in the recurrences (26)(27) are positive thanks to (H1)-(H2), also implies that $p_{\mathbf{n}, \mathbf{m}}^{\ell}$ and $C_{\mathbf{n}}^{\ell}$ can only vanish if they vanish for $\ell-1$ and, by induction, at $\ell=0$.

Now, for the $\ell^{\infty}$ estimate, notice that

$$
\begin{array}{r}
\left(v_{1, m_{1}}^{-}+v_{1, m_{1}}^{+}+v_{2, m_{2}}^{-}+v_{2, m_{2}}^{+}\right) \frac{\delta t}{\delta x}+\beta\left(F_{1, \mathbf{n}}^{\ell,-}+F_{1, \mathbf{n}}^{\ell,+}+F_{2, \mathbf{n}}^{\ell,-}+F_{2, \mathbf{n}}^{\ell,+}\right) \frac{\delta t}{\delta v}+ \\
\beta\left(v_{1, m_{1}}^{-}+v_{1, m_{1}}^{+}+v_{2, m_{2}}^{-}+v_{2, m_{2}}^{+}\right) \frac{\delta t}{\delta v}+B_{\mathbf{n}, \mathbf{m}}^{\ell}+2 \beta \frac{\delta t}{\delta v^{2}}=\left(1-\delta t b_{\mathbf{n}, \mathbf{m}}^{\ell}\right), \\
E_{\mathbf{n}}^{\ell}+4 \frac{\delta t}{\delta x^{2}} \kappa=\left(1-\delta t e_{\mathbf{n}}^{\ell}\right) .
\end{array}
$$

Let us assume first that the boundary data vanish. Using $0 \leq p_{\mathbf{n}^{\prime}, \mathbf{m}^{\prime}}^{\ell} \leq\left\|p_{\mathbf{n}, \mathbf{m}}^{\ell}\right\|_{\infty}$ and $0 \leq C_{\mathbf{n}^{\prime}}^{\ell} \leq\left\|C_{\mathbf{n}}^{\ell}\right\|_{\infty}$ for all $\mathbf{n}^{\prime}, \mathbf{m}^{\prime}$ in (26)-(27), identities (38)-(39) imply the $\ell^{\infty}$ bounds $\left\|p_{\mathbf{n}, \mathbf{m}}^{\ell+1}\right\|_{\infty} \leq\left\|p_{\mathbf{n}, \mathbf{m}}^{\ell}\right\|_{\infty} \leq\left\|p_{\mathbf{n}, \mathbf{m}}^{0}\right\|_{\infty}$ and $\left\|C_{\mathbf{n}}^{\ell+1}\right\|_{\infty} \leq\left\|C_{\mathbf{n}}^{\ell}\right\|_{\infty} \leq\left\|C_{\mathbf{n}}^{0}\right\|_{\infty}$, for $\ell \geq 0$ thanks to (H3). The contractivity property follows from the positivity of $p_{\mathbf{n}, \mathbf{m}}^{\ell}$, in view of (38)-(39) and (28).

Let us consider now non zero boundary data and fix the coefficients in (26), so that it becomes a linear recurrence. Then, $\tilde{p}_{\mathbf{n}, \mathbf{m}}^{\ell}=-p_{\mathbf{n}, \mathbf{m}}^{\ell}$ is a solution of (26) with initial and boundary data $-p_{\mathbf{n}, \mathbf{m}}^{0}$ and $-g_{\mathbf{n}, \mathbf{m}}^{\ell}$. In a similar way, $\tilde{p}_{\mathbf{n}, \mathbf{m}}^{\ell}=Z$ is a solution of (26) with initial and boundary data $Z$ and an additional right hand side $\delta t b_{\mathbf{n}, \mathbf{m}}^{\ell} Z$. Set now $Z=\operatorname{Max}_{\mathbf{n}, \mathbf{m}}\left|p_{\mathbf{n}, \mathbf{m}}^{0}\right|+\operatorname{Max}_{j, \mathbf{n}, \mathbf{m}}\left|g_{\mathbf{n}, \mathbf{m}}^{j}\right|$. We see that $\tilde{p}_{\mathbf{n}, \mathbf{m}}^{\ell}=-p_{\mathbf{n}, \mathbf{m}}^{\ell}+Z$ is a solution of (26) with nonnegative initial and boundary data, and a positive source. By induction on $\ell$, we prove that $-p_{\mathbf{n}, \mathbf{m}}^{\ell}+Z \geq 0$, thus, $0 \leq p_{\mathbf{n}, \mathbf{m}}^{\ell} \leq Z$. This yields the desired $\ell^{\infty}$ estimate on $p_{\mathbf{n}, \mathbf{m}}^{\ell}$. Notice that to estimate $p_{\mathbf{n}, \mathbf{m}}^{j}$ up to the time level $\ell$ only the values of the boundary condition up to the time level $\ell-1$ are used.

We turn now to (27). Since $e_{\mathbf{n}}^{\ell} C_{\mathbf{n}}^{\ell} \geq 0$, we see by induction that $C_{\mathbf{n}}^{\ell} \leq \tilde{C}_{\mathbf{n}}^{\ell}$, $\tilde{C}_{\mathbf{n}}^{\ell}$ being the solution of (27) with $e_{\mathbf{n}}^{\ell}=0$, that is,

$$
\tilde{C}_{\mathbf{n}}^{\ell+1}=\left[1-4 \frac{\delta t}{\delta x^{2}} \kappa\right] \tilde{C}_{\mathbf{n}}^{\ell}+\frac{\delta t}{\delta x^{2}} \kappa\left[\tilde{C}_{n_{1}+1, n_{2}}^{\ell}+\tilde{C}_{n_{1}-1, n_{2}}^{\ell}+\tilde{C}_{n_{1}, n_{2}+1}^{\ell}+\tilde{C}_{n_{1}, n_{2}-1}^{\ell}\right]
$$

keeping the same initial and boundary data. We split $\tilde{C}_{\mathbf{n}}^{\ell}=\tilde{C}_{\mathbf{n}}^{1, \ell}+\tilde{C}_{\mathbf{n}}^{2, \ell}$, where $\tilde{C}_{\mathbf{n}}^{1, \ell}$ collects the contribution of the initial datum with zero boundary conditions and $\tilde{C}_{\mathbf{n}}^{2, \ell}$ collects the contribution of the boundary condition $d_{\mathbf{n}}^{\ell}$ with zero initial datum. As before, by induction on $\ell, 0 \leq \tilde{C}_{\mathbf{n}}^{1, \ell} \leq\left\|C_{\mathbf{n}}^{0}\right\|_{\infty}$. Now, we can recast the equations defining $\tilde{C}_{\mathbf{n}}^{2, \ell}$ in the matrix form (34) with $\mathbf{B}_{2}^{\ell}=0, \mathbf{A}_{2}=\mathcal{I}+\kappa \frac{\delta t}{\delta x^{2}} \mathcal{N}$ 
and $\tilde{Y}^{\ell}$ given by (35)-(36). Thanks to (H2), the sums of the absolute values of the elements of the rows of $\mathbf{A}_{2}$ are bounded by 1. Thus, $\left\|\mathbf{A}_{2}\right\|_{\infty} \leq 1$. For Dirichlet and Neumann data generated from a smooth bounded function $\tilde{d}(\mathbf{x}, t)$, that is, $d_{\mathbf{n}}^{\ell}=\tilde{d}\left(\mathbf{x}_{\mathbf{n}}, t_{\ell}\right)$ at $n_{2}=0, N_{2}$ and $d_{\mathbf{n}}^{\ell}= \pm \frac{\partial \tilde{d}}{\partial x_{1}}\left(\mathbf{x}_{\mathbf{n}}, t_{\ell}\right)$ at $n_{1}=N_{1}, 0$, we set $\tilde{d}_{\mathbf{n}}^{\ell}=\tilde{d}\left(\mathbf{x}_{\mathbf{n}}, t_{\ell}\right)$ and $\tilde{C}_{\mathbf{n}}^{2, \ell}=\tilde{C}_{\mathbf{n}}^{3, \ell}+\tilde{d}_{\mathbf{n}}^{\ell}$. Then, $\tilde{C}_{\mathbf{n}}^{3, \ell}$ satisfies

$$
\tilde{C}_{\mathbf{n}}^{3, \ell+1}=\mathbf{A}_{2} \tilde{C}_{\mathbf{n}}^{3, \ell}+\tilde{H}^{\ell}=\left(\mathbf{A}_{2}\right)^{\ell+1} \tilde{C}_{\mathbf{n}}^{3,0}+\sum_{j=0}^{\ell}\left(\mathbf{A}_{2}\right)^{j} \tilde{H}^{\ell-j},
$$

where $\tilde{C}_{\mathbf{n}}^{3,0}=\tilde{C}_{\mathbf{n}}^{2,0}-\tilde{d}_{\mathbf{n}}^{0}$ and

$$
\begin{aligned}
& \tilde{H}^{\ell}=\left(\tilde{h}_{1,1}^{\ell}, \ldots, \tilde{h}_{N_{1}-1,1}^{\ell}, \ldots, \tilde{h}_{1, j}^{\ell}, \ldots, \tilde{h}_{N_{1}-1, j}^{\ell} \ldots, \tilde{h}_{1, N_{2}-1}^{\ell}, \ldots, \tilde{h}_{N_{1}-1, N_{2}-1}^{\ell}\right), \\
& \tilde{h}_{\mathbf{n}}^{\ell}=\delta t \frac{\tilde{d}_{\mathbf{n}}^{\ell}-\tilde{d}_{\mathbf{n}}^{\ell+1}}{\delta t}+\frac{\delta t}{\delta x^{2}} \kappa\left[\tilde{d}_{n_{1}+1, n_{2}}^{\ell}+\tilde{d}_{n_{1}-1, n_{2}}^{\ell}-4 \tilde{d}_{\mathbf{n}}^{\ell}+\tilde{d}_{n_{1}, n_{2}+1}^{\ell}+\tilde{d}_{n_{1}, n_{2}-1}^{\ell}\right] .
\end{aligned}
$$

Now, Taylor expansions for the smooth function $\tilde{d}$ show that $\tilde{h}_{\mathbf{n}}^{\ell}=\delta t\left(\tilde{h}_{\mathbf{n}}^{1, \ell}+\tilde{h}_{\mathbf{n}}^{2, \ell}\right)$, with bounded sequences $\tilde{h}_{\mathbf{n}}^{i, \ell}$. Since $\left\|\mathbf{A}_{2}\right\|_{\infty} \leq 1$, identity (41) implies

$$
\begin{aligned}
\left\|\tilde{C}_{\mathbf{n}}^{3, \ell+1}\right\|_{\infty} & \leq\left\|\tilde{C}_{\mathbf{n}}^{3,0}\right\|_{\infty}+\sum_{j=0}^{\ell}\left\|\tilde{h}_{\mathbf{n}}^{j}\right\|_{\infty} \\
& \leq\left\|\tilde{d}_{\mathbf{n}}^{0}\right\|_{\infty}+2 T \operatorname{Max}_{j \leq \ell}\left[\left\|\tilde{h}_{\mathbf{n}}^{1, \ell}\right\|_{\infty},\left\|\tilde{h}_{\mathbf{n}}^{2, \ell}\right\|_{\infty}\right]=K_{0}(T),
\end{aligned}
$$

because $\delta t(\ell+1) \leq \delta t \mathcal{L} \leq T$. Together with the previous estimate on $\left\|\tilde{C}_{\mathbf{n}}^{1, \ell}\right\|_{\infty}$, this yields the desired bound on $\left\|C_{\mathbf{n}}^{\ell}\right\|_{\infty}$ in terms of $f\left(d_{\mathbf{n}}^{j}, T\right)=\operatorname{Max}_{j \leq \ell-1}\left\|\tilde{d}_{\mathbf{n}}^{j}\right\|_{\infty}+$ $K_{0}(T)$. Notice that for data of the form (32), and the parameters in Section 3, $\tilde{d}_{\mathbf{n}}^{\ell}$ can be approximately generated using the function $c_{L}(t) e^{-a^{2} x_{2}^{2}} x_{1}^{2}$ in the computational region and $\sum_{j=1}^{\mathcal{L}}\left\|\tilde{h}_{\mathbf{n}}^{j}\right\|_{\infty}$ is bounded in terms of $\int_{0}^{T}\left|c_{L}^{\prime}(s)\right| d s+$ $2 \kappa\left(2 a^{4}+a^{2}+1\right) \int_{0}^{T} c_{L}(s) d s$. In general, for data generated from smooth functions, we may use $\tilde{d}\left(\mathbf{x}_{\mathbf{n}}, t_{\ell}\right), \tilde{d}$ being the smooth solution of the associated continuous Neumann-Dirichlet problem.

Finally, when we consider the boundary condition (30)-(31) for $p_{\mathbf{n}, \mathbf{m}}^{\ell}$, solutions are still nonnegative if the initial data are nonnegative because $S_{0}^{d}$ and $S_{L}^{d}$ are positive operators.

Notice that Proposition 2.1 holds regardless of the definition of the force field, whether it is fixed and known or it depends on the concentrations. Let us obtain now conditions ensuring that (H1), (H2) and (H3) are verified.

Proposition 2.2. Under the hypotheses (I1), (I2), (H0) of Proposition 2.1, conditions (H1),(H2),(H3) hold when $0 \leq \ell \leq \mathcal{L}$ for solutions of the scheme (26)-(27) with force field (29) provided they hold for $\ell=0$ and $\delta t, \delta x, \delta v$, $\frac{\delta t}{\delta x^{2}}, \frac{\delta t}{\delta x \delta v}$ and $\frac{\delta t}{\delta v^{2}}$ are sufficiently small, depending on the continuous model parameters and the initial and boundary data, as well as on $T$ and $V$.

When the force field is given by a known field, that is, $\mathbf{F}_{\mathbf{n}}^{\ell}=\mathbf{F}\left(\mathbf{x}_{\mathbf{n}}, t_{\ell}\right)$, this result still holds, replacing the dependence of the step size on the initial and boundary data for the concentrations by dependence on $\|\mathbf{F}\|_{L_{\mathbf{x}, t}^{\infty}}$. 
Proof. Notice that $\frac{\delta t}{\delta x}$ and $\frac{\delta t}{\delta v}$ can be made as small as wished by taking $\delta x, \delta v, \frac{\delta t}{\delta x^{2}}$ and $\frac{\delta t}{\delta v^{2}}$ small enough, and $\frac{\delta t}{\delta x \delta v}$ too, provided we keep the ratio $\frac{\delta v}{\delta x}$ constant.

Let us first assume that the boundary data vanish. Revisiting the proof of Proposition 2.1 we see that the positivity and uniform $\ell^{\infty}$ bounds hold when $\ell=1$. Let us now check that (H1), (H2) and (H3) also hold for $\ell=1$. Once we know that $p_{\mathbf{n}, \mathbf{m}}^{\ell} \geq 0$ for $\ell=0,1,(\mathrm{H} 3)$ is immediate for $\ell=1$ provided (H0) holds. Now, we observe that

$$
\delta v^{2} \sum_{\mathbf{m}^{\prime}} a_{\mathbf{m}^{\prime}} w\left(v_{\mathbf{m}^{\prime}}\right) p_{\mathbf{n}, \mathbf{m}^{\prime}}^{1} e^{\lambda t_{1}} \leq\left\|p_{\mathbf{n}, \mathbf{m}}^{0}\right\|_{\infty} e^{\lambda T} \delta v^{2} \sum_{\mathbf{m}^{\prime}} a_{\mathbf{m}^{\prime}} w\left(v_{\mathbf{m}^{\prime}}\right) \sim K_{1}(T) .
$$

Thus, (H2) holds provided $\delta t$ and $\delta t / \delta x^{2}$ are small enough depending on $\chi, \kappa, \nu$, and $K_{1}(T)$, but not on $\ell$. For (H1) we need to control uniformly $\sum_{\mathbf{m}} p_{\mathbf{n}, \mathbf{m}}^{1}$ and $\delta x \mathbf{F}_{\mathbf{n}}^{1}$, that is, $C_{n_{1}+1, n_{2}}^{1}-C_{n_{1}, n_{2}}^{1}$ and $C_{n_{1}, n_{2}+1}^{1}-C_{n_{1}, n_{2}}^{1}$. These later magnitudes are bounded by $2\left\|C_{\mathbf{n}}^{0}\right\|_{\infty}$ thanks to the $\ell^{\infty}$ estimate. Regarding $\sum_{\mathbf{m}} p_{\mathbf{n}, \mathbf{m}}^{1}$ we observe that:

$$
\delta t \delta v^{2} \sum_{k=0}^{1} \sum_{\mathbf{m}^{\prime}} e^{\lambda t_{k}} a_{\mathbf{m}^{\prime}} p_{\mathbf{n}, \mathbf{m}^{\prime}}^{k} \leq \delta t \delta v^{2} \tilde{a} e^{\lambda T} \sum_{k=0}^{1} \sum_{\mathbf{m}^{\prime}} p_{\mathbf{n}, \mathbf{m}^{\prime}}^{k} .
$$

A discrete version of the inequality established in [18]:

$$
\left\|\int_{\mathbb{R}^{N}} p d \mathbf{v}\right\|_{L_{\mathbf{x}}^{\infty}} \leq C_{N, \mu}\|p\|_{L_{\mathbf{x}}^{\infty}}^{1-N / \mu}\left\|\left(1+|\mathbf{v}|^{2}\right)^{\mu / 2} p\right\|_{L_{\mathbf{x} \mathbf{v}}^{\infty}}^{N / \mu}, \quad \mu>N,
$$

applied to $p_{\mathbf{n}, \mathbf{m}}^{k}$ yields for (42) the upper bound

$$
\delta t e^{\lambda T} \tilde{a} C_{N, \mu}\left(1+V^{2}\right)^{\mu / 2} \sum_{k=0}^{1}\left\|p_{\mathbf{n}, \mathbf{m}}^{k}\right\|_{\infty} \leq T e^{\lambda T} \tilde{a} C_{N, \mu}\left(1+V^{2}\right)^{\mu / 2}\left\|p_{\mathbf{n}, \mathbf{m}}^{0}\right\|_{\infty},
$$

or even simpler,

$$
\delta t \delta v^{2} \tilde{a} e^{\lambda T} \sum_{k=0}^{1} \sum_{\mathbf{m}^{\prime}} p_{\mathbf{n}, \mathbf{m}^{\prime}}^{k} \leq 4 T \tilde{a} e^{\lambda T} V^{2}\left\|p_{\mathbf{n}, \mathbf{m}}^{0}\right\|_{\infty},
$$

taking into account that $\ell \delta t \leq \mathcal{L} \delta t=T$ and that $M \delta v=2 V$ when the velocities belong to $[-V, V]^{2}$ and the time lies in $[0, T]$. We conclude that for $\frac{\delta t}{\delta x^{2}}, \frac{\delta t}{\delta v^{2}}$, $\frac{\delta t}{\delta v \delta x} \delta t, \delta x, \delta v$, small enough, depending on the involved parameters and the initial data, (H1) holds when $\ell=1$.

By induction, assuming (H1), (H2) and (H3) hold for $\ell \leq \mathcal{L}-1$, we have positivity and $\ell^{\infty}$ estimates up to $\ell=\mathcal{L}$ by Proposition 2.1. Thus, we can see that (H1), (H2) and (H3) hold for $\mathcal{L}$ in a similar way.

Let us now set $d_{\mathbf{n}}^{\ell} \neq 0$. The positivity and $\ell^{\infty}$ estimates hold for $\ell=1$. The only change in the previous arguments to prove that (H1), (H2), (H3) hold for $\ell=1$ first, and for $\ell=2, \ldots \mathcal{L}$ later, comes from the uniform bound on $\delta x \mathbf{F}_{\mathbf{n}}^{\ell}$, which now involves $2\left\|C_{\mathbf{n}}^{0}\right\|_{\infty}+2 f\left(d_{\mathbf{n}}^{j}, T\right)$.

Finally, we also set $g_{\mathbf{n}, \mathbf{m}}^{\ell} \neq 0$. The only additional change in the previous arguments is that $\left\|p_{\mathbf{n}, \mathbf{m}}^{0}\right\|_{\infty}$ is replaced by $\left\|p_{\mathbf{n}, \mathbf{m}}^{0}\right\|_{\infty}+\operatorname{Max}_{j}\left\|g_{\mathbf{n}, \mathbf{m}}^{j}\right\|_{\infty}$.

When $\mathbf{F}_{\mathbf{n}}^{\ell}$ represents a known force field, we have to estimate the term $\frac{\delta t}{\delta v} \mathbf{F}\left(\mathbf{x}_{\mathbf{n}}, t_{\ell}\right)$ instead of $\frac{\delta t}{\delta v \delta x} h\left(C_{\mathbf{n}}^{\ell}\right) e^{\nu t_{\ell}}\left(C_{n_{1}+1, n_{2}}^{\ell}-C_{n_{1}, n_{2}}^{\ell}, C_{n_{1}, n_{2}+1}^{\ell}-C_{n_{1}, n_{2}}^{\ell}\right)$, which is simply done using $\|\mathbf{F}\|_{L_{\mathbf{x}, t}^{\infty}}$. 
In addition, the following stability estimate holds:

Proposition 2.3. Under the hypotheses of Proposition 2.2, and assuming $p_{\mathbf{n}, \mathbf{m}}^{\ell} \geq 0, g_{\mathbf{n}, \mathbf{m}}^{\ell}=0$ for all $\mathbf{m}, \mathbf{n}$, and $\ell \leq \mathcal{L}$, we have

$$
\delta x^{2} \delta v^{2} \sum_{n_{1}=1}^{N_{1}-1} \sum_{n_{2}=1}^{N_{2}-1} \sum_{m_{1}, m_{2}=1}^{M-1} p_{\mathbf{n}, \mathbf{m}}^{\ell} \leq \delta x^{2} \delta v^{2} \sum_{n_{1}=1}^{N_{1}-1} \sum_{n_{2}=1}^{N_{2}-1} \sum_{m_{1}, m_{2}=1}^{M-1} p_{\mathbf{n}, \mathbf{m}}^{0}
$$

for all $\ell \leq \mathcal{L}$. When $g_{\mathbf{n}, \mathbf{m}}^{\ell} \neq 0$ an additional term involving $g_{\mathbf{n}, \mathbf{m}}^{\ell}$ appears on the right hand side

$V \delta t \delta x \delta v^{2} \sum_{k=0}^{\ell-1} \sum_{m_{1}, m_{2}=1}^{M-1}\left[\sum_{n_{2}=1}^{N_{2}-1}\left(g_{N_{1}, n_{2}, \mathbf{m}}^{k}+g_{0, n_{2}, \mathbf{m}}^{k}\right)+\sum_{n_{1}=1}^{N_{1}-1}\left(g_{n_{1}, N_{2}, \mathbf{m}}^{k}+g_{n_{1}, 0, \mathbf{m}}^{k}\right)\right]$.

Proof. The hypotheses ensure that $b_{\mathbf{n}, \mathbf{m}}^{\ell} \geq 0$ for all $\mathbf{n}$ and $\ell \leq \mathcal{L}$. We argue by induction noticing the following. Summing over $\mathbf{m}$ in expression (26) we find that:

$$
\begin{aligned}
& \sum_{\mathbf{m}} p_{\mathbf{n}, \mathbf{m}}^{\ell+1} \leq \sum_{\mathbf{m}}\left(1-\delta t b_{\mathbf{n}, \mathbf{m}}^{\ell}\right) p_{\mathbf{n}, \mathbf{m}}^{\ell}-\frac{\delta t}{\delta x} \sum_{\mathbf{m}}\left(v_{1, m_{1}}^{-}+v_{1, m_{1}}^{+}+v_{2, m_{2}}^{-}+v_{2, m_{2}}^{+}\right) p_{\mathbf{n}, \mathbf{m}}^{\ell}+ \\
& \frac{\delta t}{\delta x} \sum_{\mathbf{m}}\left[v_{1, m_{1}}^{-} p_{n_{1}+1, n_{2}, \mathbf{m}}^{\ell}+v_{1, m_{1}}^{+} p_{n_{1}-1, n_{2}, \mathbf{m}}^{\ell}+v_{2, m_{2}}^{-} p_{n_{1}, n_{2}+1, \mathbf{m}}^{\ell}+v_{2, m_{2}}^{+} p_{n_{1}, n_{2}-1, \mathbf{m}}^{\ell}\right]
\end{aligned}
$$

where we have used, taking into account the zero boundary values for $p$ at $m_{1}, m_{2}=0$ and $m_{1}, m_{2}=M$ :

- For the sums of $p_{\mathbf{n}, m_{1} \pm 1, m_{2} \pm 1}$ :

$$
\begin{aligned}
& \frac{\delta t}{\delta v^{2}} \frac{\beta}{2} \sum_{m_{1}=1}^{M-1} \sum_{m_{2}} p_{\mathbf{n}, m_{1}+1, m_{2}}^{\ell}=\frac{\delta t}{\delta v^{2}} \frac{\beta}{2} \sum_{m_{1}=2}^{M} \sum_{m_{2}} p_{\mathbf{n}, m_{1}, m_{2}}^{\ell} \\
& =\frac{\delta t}{\delta v^{2}} \frac{\beta}{2} \sum_{m_{1}=1}^{M-1} \sum_{m_{2}} p_{\mathbf{n}, m_{1}, m_{2}}^{\ell}-\frac{\delta t}{\delta v^{2}} \frac{\beta}{2} \sum_{m_{2}} p_{\mathbf{n}, 1, m_{2}}^{\ell},
\end{aligned}
$$

with similar identities for $p_{\mathbf{n}, m_{1}-1, m_{2}}^{\ell}, p_{\mathbf{n}, m_{1}, m_{2} \pm 1}^{\ell}, F_{1, \mathbf{n}}^{\ell, \pm} p_{\mathbf{n}, m_{1} \pm 1, m_{2}}^{\ell}$ and $F_{2, \mathbf{n}}^{\ell, \pm} p_{\mathbf{n}, m_{1}, m_{2} \pm 1}^{\ell}$.

- For the sums involving $v_{1, m_{1}}^{ \pm} p_{\mathbf{n}, m_{1} \pm 1, m_{2}}$ :

$$
\begin{aligned}
& \frac{\delta t}{\delta v} \beta \sum_{m_{1}=1}^{M-1} \sum_{m_{2}} v_{1, m_{1}}^{+}\left(p_{\mathbf{n}, m_{1}+1, m_{2}}^{\ell}-p_{\mathbf{n}, m_{1}, m_{2}}^{\ell}\right)= \\
& \frac{\delta t}{\delta v} \beta \sum_{m_{1}=1}^{M-1} \sum_{m_{2}}\left(v_{1, m_{1}-1}^{+}-v_{1, m_{1}}^{+}\right) p_{\mathbf{n}, m_{1}, m_{2}}^{\ell}-\frac{\delta t}{\delta v} \beta v_{1,0}^{+} \sum_{m_{2}} p_{\mathbf{n}, 1, m_{2}}^{\ell}= \\
& -\delta t \beta \sum_{m_{1}=M_{0}+1}^{M-1} \sum_{m_{2}} p_{\mathbf{n}, m_{1}, m_{2}}^{\ell}-\frac{\delta t}{\delta v} \beta v_{1,0}^{+} \sum_{m_{2}} p_{\mathbf{n}, 1, m_{2}}^{\ell} .
\end{aligned}
$$

Analogous identities hold for $v_{1, m_{1}}^{-} p_{\mathbf{n}, m_{1}-1, m_{2}} v_{2, m_{2}}^{ \pm} p_{\mathbf{n}, m_{1}, m_{2} \pm 1}$.

Summing now with respect to $\mathbf{n}$, we find

$$
\sum_{\mathbf{n}, \mathbf{m}} p_{\mathbf{n}, \mathbf{m}}^{\ell+1} \leq \sum_{\mathbf{n}, \mathbf{m}}\left(1-\delta t b_{\mathbf{n}, \mathbf{m}}^{\ell}\right) p_{\mathbf{n}, \mathbf{m}}^{\ell},
$$


where we have used

$$
\begin{aligned}
\sum_{n_{1}=1}^{N_{1}-1} \sum_{n_{2}, \mathbf{m}} v_{1, m_{1}}^{-} p_{n_{1}+1, n_{2}, \mathbf{m}}^{\ell}= & \sum_{n_{1}=1}^{N_{1}-1} \sum_{n_{2}, \mathbf{m}} v_{1, m_{1}}^{-} p_{n_{1}, n_{2}, \mathbf{m}}^{\ell}+ \\
& \sum_{n_{2}, \mathbf{m}}\left[v_{1, m_{1}}^{-} p_{N_{1}, n_{2}, \mathbf{m}}^{\ell}-v_{1, m_{1}}^{-} p_{1, n_{2}, \mathbf{m}}^{\ell}\right], \\
\sum_{n_{1}=1}^{N_{1}-1} \sum_{n_{2}, \mathbf{m}} v_{1, m_{1}}^{+} p_{n_{1}-1, n_{2}, \mathbf{m}}^{\ell}= & \sum_{n_{1}=1}^{N_{1}-1} \sum_{n_{2}, \mathbf{m}} v_{1, m_{1}}^{+} p_{n_{1}, n_{2}, \mathbf{m}}^{\ell}+ \\
& \sum_{n_{2}, \mathbf{m}}\left[v_{1, m_{1}}^{+} p_{0, n_{2}, \mathbf{m}}^{\ell}-v_{1, m_{1}}^{+} p_{N_{1}-1, n_{2}, \mathbf{m}}^{\ell}\right],
\end{aligned}
$$

and $p_{0, n_{2}, \mathbf{m}}^{\ell}=0$ when $v_{1, m_{1}}>0, p_{N_{1}, n_{2}, \mathbf{m}}^{\ell}=0$ when $v_{1, m_{1}}<0$. Analogous identities hold for $v_{2, m_{2}}^{ \pm} p_{n_{1}, n_{2} \pm 1, \mathbf{m}}$.

Inequality (44) implies:

$$
\sum_{\mathbf{n}, \mathbf{m}} p_{\mathbf{n}, \mathbf{m}}^{\ell+1} \leq \sum_{\mathbf{n}, \mathbf{m}} p_{\mathbf{n}, \mathbf{m}}^{\ell},
$$

if $p_{\mathbf{n}, \mathbf{m}}^{k} \geq 0$ for $k \leq \ell$ and provided (H0) holds, in view of

$$
\begin{aligned}
\sum_{\mathbf{n}, \mathbf{m}} p_{\mathbf{n}, \mathbf{m}}^{\ell+1} & \leq\left(1+\left[\max \left(\alpha \delta_{\mathbf{v}^{0}}\right)+2 \beta-\lambda\right] \delta t\right)^{\ell+1} \sum_{\mathbf{n}, \mathbf{m}} p_{\mathbf{n}, \mathbf{m}}^{0} \\
& \leq e^{\left[\max \left(\alpha \delta_{\mathbf{v}} 0\right)+2 \beta-\lambda\right] T} \sum_{\mathbf{n}, \mathbf{m}} p_{\mathbf{n}, \mathbf{m}}^{0} .
\end{aligned}
$$

When $p_{0, n_{2}, \mathbf{m}}^{\ell}=g_{0, n_{2}, \mathbf{m}}^{\ell}$ for $v_{1, m_{1}}>0$ and $p_{N_{1}, n_{2}, \mathbf{m}}^{\ell}=g_{0, n_{2}, \mathbf{m}}^{\ell}$ for $v_{1, m_{1}}<0$, we have additional terms of the form

$$
\sum_{n_{2}, m_{2}}\left[\sum_{m_{1}=1}^{M_{0}} v_{1, m_{1}}^{-} g_{N_{1}, n_{2}, \mathbf{m}}^{\ell}+\sum_{m_{1}=M_{0}+1}^{M-1} v_{1, m_{1}}^{+} g_{0, n_{2}, \mathbf{m}}^{\ell}\right] \leq V \sum_{n_{2}, \mathbf{m}}\left[g_{N_{1}, n_{2}, \mathbf{m}}^{\ell}+g_{0, n_{2}, \mathbf{m}}^{\ell}\right]
$$

multiplied by $\frac{\delta t}{\delta x}$ in (44). Iterating the inequality, we get (46) with an extra term

$$
\begin{aligned}
& V \frac{\delta t}{\delta x} \sum_{k=0}^{\ell}\left(1+\left[\max \left(\alpha \delta_{\mathbf{v}^{0}}\right)+2 \beta-\lambda\right] \delta t\right)^{\ell-k} \sum_{n_{2}, \mathbf{m}}\left[g_{N_{1}, n_{2}, \mathbf{m}}^{\ell}+g_{0, n_{2}, \mathbf{m}}^{k}\right] \\
& \leq V \frac{\delta t}{\delta x} \sum_{k=0}^{\ell} \sum_{n_{2}, \mathbf{m}}\left[g_{N_{1}, n_{2}, \mathbf{m}}^{k}+g_{0, n_{2}, \mathbf{m}}^{k}\right]
\end{aligned}
$$

applying (H0). Similar bounds hold on the walls $n_{2}=0, N_{2}$.

After these preliminary stability estimates we establish now a convergence result. Existence of nonnegative weak solutions for problems of the form (1)-(8), (13) has been shown under the conditions [14]:

- $p_{0}, C_{0}, g, d \geq 0$,

- $C_{0} \in W^{2, \infty}(\Omega)$,

- $\left(1+|\mathbf{v}|^{2}\right)^{\mu / 2} p_{0} \in L^{\infty} \cap L^{1}\left(\Omega \times \mathbb{R}^{2}\right), \mu>2$,

- $d \in L^{\infty}\left(0, T ; L^{\infty}(\partial \Omega)\right)$,

- $(1+|\mathbf{v} \cdot \mathbf{n}|)\left(1+|\mathbf{v}|^{2}\right)^{\mu / 2} g \in L^{\infty}\left(0, T ; L^{\infty} \cap L^{1}\left(\Gamma^{-}\right)\right)$, 
for bounded $\Omega \subset \mathbb{R}^{2}$, with either fixed bounded fields $\mathbf{F}$ or nonlinear fields $\mathbf{F}(C)$ given by (4). Ref. [14] considered annular domains, but the proof extends to other domains for which optimal decay estimates of the gradients of heat kernels are available, such as slabs. Considering the boundary conditions (9)(10) a solution exists under additional conditions on the parameters involved in them [14]. Such solutions are in principle defined almost everywhere. Increasing the regularity assumptions of the data we obtain more regular solutions.

We next prove convergence of the scheme to smooth solutions under certain hypotheses. Notice that the convergence of the scheme entails somehow uniqueness of smooth enough nonnegative solutions since similar arguments imply that the error when comparing two smooth nonnegative solutions tends to zero. The solutions we consider are smooth enough for the truncation error generated when introducing them in the scheme to involve powers of $\delta t, \delta x$ and $\delta v$ multiplied by bounded coefficients.

Theorem 2.4. Let us select $X, V, T>0$ and consider a smooth nonnegative solution $p \in C^{2}\left(\bar{\Omega}_{\mathbf{x}} \times[0, T] ; C^{4}\left(\bar{\Omega}_{\mathbf{v}}\right)\right), c \in C^{2}\left([0, T] ; C^{4}\left(\bar{\Omega}_{\mathbf{x}}\right)\right)$ of the problem (16)-(18), with initial data $p_{0} \geq 0$ and $c_{0} \geq 0$, as well as nonnegative boundary conditions of the form (13) on $\partial \Omega_{\mathbf{x}}$ and zero boundary conditions on $\partial \Omega_{\mathbf{v}}$ for $p$, together with nonnegative Dirichlet boundary conditions at $x_{2}= \pm \tau X$ and boundary conditions of the form (8) at $x_{1}=0, X$ for $c$. We assume that either $\mathbf{F}(c)$ is a smooth enough function of $c$, bounded and with bounded derivatives, or it is a fixed and known smooth field. Then, the solutions of the scheme (26)-(27) converge to $(p, c)$ as the discretization steps $\delta x, \delta v$ and $\delta t$ tend to zero under the hypotheses of Propositions 2.1-2.2 and the scheme is of order one.

Proof. Let $q_{\mathbf{n}, \mathbf{m}}^{\ell}=p\left(\mathbf{x}_{\mathbf{n}}, \mathbf{v}_{\mathbf{m}}, t_{\ell}\right) \geq 0$ and $s_{\mathbf{n}}^{\ell}=c\left(\mathbf{x}_{\mathbf{n}}, t_{\ell}\right) \geq 0$ be the solutions of the continuous problem evaluated at the grid points. They fulfill the difference scheme (26)-(27) for $n_{i}=1, \ldots, N_{i}-1, m_{i}=1, \ldots, M-1, i=1,2, \ell=0, \ldots, \mathcal{L}-1$, with boundary conditions of the form (I2) defined from (32) and a right hand side representing the truncation error multiplied by $\delta t: z_{\mathbf{n}, \mathbf{m}}^{\ell}=\delta t\left(z_{\mathbf{n}, \mathbf{m}}^{1, \ell} \delta t+\right.$ $\left.z_{\mathbf{n}, \mathbf{m}}^{2, \ell} \delta x+z_{\mathbf{n}, \mathbf{m}}^{3, \ell} \delta v\right)$ and $y_{\mathbf{n}}^{\ell}=\delta t\left(y_{\mathbf{n}}^{1, \ell} \delta t+y_{\mathbf{n}}^{2, \ell} \delta x+y_{\mathbf{n}}^{3, \ell} \delta v^{4}\right)$, where the coefficients $z_{\mathbf{n}, \mathbf{m}}^{i, \ell}$ and $y_{\mathbf{n}}^{i, \ell}, i=1,2,3$, are bounded. Notice that the integral with respect to $\mathbf{v}$ in equation (2) contributes an error term with respect to $\mathbf{v}$.

Let $p_{\mathbf{n}, \mathbf{m}}^{\ell}$ and $c_{\mathbf{n}}^{\ell}$ be the solutions of scheme (26)-(27) with the same initial conditions and the same boundary conditions of the form (I2). We will use the matrix version of these equations, that is, system (33)-(34). Relabelling in a similar way, we recast the equations for $q_{\mathbf{n}, \mathbf{m}}^{\ell}$ and $s_{\mathbf{n}}^{\ell}$ as a nonlinear system:

$$
\begin{array}{r}
Q^{\ell+1}=\mathbf{A}_{1} Q^{\ell}+\operatorname{diag}\left[\sum_{k=0}^{\ell} \mathbf{B}_{1}^{k} Q^{k}+D_{1}^{\ell}\left(S^{\ell}\right)\right] Q^{\ell}+\mathbf{D}_{2}^{\ell}\left(S^{\ell}\right) Q^{\ell}+\tilde{Z}^{\ell}+Z^{\ell}, \\
S^{\ell+1}=\mathbf{A}_{2} S^{\ell}+\operatorname{diag}\left[\mathbf{B}_{2}^{\ell} Q^{\ell}\right] S^{\ell}+\tilde{Y}^{\ell}+Y^{\ell}
\end{array}
$$

where 'diag' stands for diagonal matrix and the matrices $\mathbf{A}_{i}, \mathbf{B}_{i}^{\ell}, i=1,2$, and $\operatorname{diag}\left(D_{1}^{\ell}\right), \mathbf{D}_{2}^{\ell}$ are defined as in (33)-(34). The contribution from the initial and boundary data is exactly the same: $\tilde{Z}^{\ell}$ and $\tilde{Y}^{\ell}$. Besides, additional source terms 
$Z^{\ell}$ and $Y^{\ell}$ appear in the right hand side that collect the truncation error. Substracting $U^{\ell}=P^{\ell}-Q^{\ell}$ and $R^{\ell}=C^{\ell}-S^{\ell}$ we find:

$$
\begin{aligned}
U^{\ell+1}= & \tilde{\mathbf{A}}_{1}^{\ell} U^{\ell}+\operatorname{diag}\left[\sum_{k=0}^{\ell} \mathbf{B}_{1}^{k} U^{k}+\left[D_{1}^{\ell}\left(C^{\ell}\right)-D_{1}^{\ell}\left(S^{\ell}\right)\right]\right] Q^{\ell} \\
& +\left[\mathbf{D}_{2}^{\ell}\left(C^{\ell}\right)-\mathbf{D}_{2}^{\ell}\left(S^{\ell}\right)\right] Q^{\ell}-Z^{\ell}, \\
R^{\ell+1}= & \tilde{\mathbf{A}}_{2}^{\ell} R^{\ell}+\operatorname{diag}\left[\mathbf{B}_{2}^{\ell} U^{\ell}\right] S^{\ell}-Y^{\ell},
\end{aligned}
$$

where $\tilde{\mathbf{A}}_{1}^{\ell}=\mathbf{A}_{1}+\operatorname{diag}\left[\sum_{k=0}^{\ell} \mathbf{B}_{1}^{k} P^{k}+D_{1}^{\ell}\left(C^{\ell}\right)\right]+\mathbf{D}_{2}^{\ell}\left(C^{\ell}\right)$ and $\tilde{\mathbf{A}}_{2}^{\ell}=\mathbf{A}_{2}+$ $\operatorname{diag}\left[\mathbf{B}_{2}^{k} P^{\ell}\right]$. The components $U_{\zeta}^{\ell}$ and $R_{\xi}^{\ell}$ of of the vectors $U^{\ell}$ and $R^{\ell}$ correspond to the relabeled sequences $u_{\mathbf{n}, \mathbf{m}}^{\ell}=p_{\mathbf{n}, \mathbf{m}}^{\ell}-q_{\mathbf{n}, \mathbf{m}}^{\ell}$ and $r_{\mathbf{n}}^{\ell}=c_{\mathbf{n}}^{\ell}-s_{\mathbf{n}}^{\ell}$. Since the initial data are the same, $U^{0}=0$ and $R^{0}=0$. For the same reason, the sources representing the boundary data cancel. Next, we estimate the size of the different matrices appearing in (49)-(50).

Let us consider first the matrices $\tilde{\mathbf{A}}_{1}^{\ell}$ and $\tilde{\mathbf{A}}_{2}^{\ell}$. Recall that for a matrix $\mathcal{M}$, $\|\mathcal{M}\|_{\infty}$ is the maximum absolute sum of the rows of the matrix. Notice that the elements of the rows of $\tilde{\mathbf{A}}_{1}^{\ell}$ are the coefficients of the discretized density in (26) and the elements of the rows of $\tilde{\mathbf{A}}_{2}^{\ell}$ are the coefficients of the discretized concentration in (27), with pertinent modifications when boundary points are involved to account for the boundary conditions. Revisiting the proofs of Propositions 2.1-2.2 we show that $p_{\mathbf{n}, \mathbf{m}}^{\ell} \geq 0, c_{\mathbf{n}}^{\ell} \geq 0$, that $p_{\mathbf{n}, \mathbf{m}}^{\ell}, c_{\mathbf{n}}^{\ell}, \delta t \delta v^{2} \sum_{k} \sum_{\mathbf{n}, \mathbf{m}} p_{\mathbf{n}, \mathbf{m}}^{k}$ are bounded in terms of the data and (H1)-(H3) are guaranteed. Notice that either known functions $\alpha$ and $\mathbf{F}$ or general functions $\alpha(c)$ and $\mathbf{F}(c)$ only enter Propositions 2.1-2.2 through their norms. Then, (38)-(39) imply estimate

$$
\left\|\tilde{\mathbf{A}}_{1}^{\ell}\right\|_{\infty}<1-\gamma \delta t<1, \quad\left\|\tilde{\mathbf{A}}_{2}^{\ell}\right\|_{\infty}<1-\gamma \delta t<1,
$$

for all $0 \leq \ell \leq \mathcal{L}$, with $\gamma=\gamma(\nu, \lambda)>0$ for $\nu>0$ and $\lambda / 2 \geq 2 \beta+\max \left|\alpha \delta_{\mathbf{v}^{0}}\right|$, when $\delta t, \delta x, \delta v, \frac{\delta t}{\delta x^{2}}$ and $\frac{\delta t}{\delta v^{2}}$ are small enough, depending on $T, V, \lambda$ and $\nu$, as well as on the norms of the initial data and the boundary data, the coefficient functions $\mathbf{F}$ and $\alpha$, and the model parameters. Notice that at rows involving boundary points the equal sign in (38)-(39) is just replaced by $\leq$.

We turn now to the nonlocal additions. The elements of $\operatorname{diag}\left[\mathbf{B}_{1}^{k} U^{k}\right] Q^{\ell}$ take the form $-\Gamma \delta t^{2} \delta v^{2} \sum_{m_{1}^{\prime}, m_{2}^{\prime}=0}^{M} e^{\lambda t_{k}} a_{\mathbf{m}^{\prime}} u_{\mathbf{n}, \mathbf{m}^{\prime}}^{k} q_{\mathbf{n m}}^{\ell}$, whereas the elements of $\operatorname{diag}\left[\mathbf{B}_{2}^{\ell} U^{\ell}\right] S^{\ell}$ are $-\chi \delta t \delta v^{2} \sum_{m_{1}^{\prime}, m_{2}^{\prime}=0}^{M} e^{\lambda t_{\ell}} a_{\mathbf{m}^{\prime}} w\left(v_{\mathbf{m}^{\prime}}\right) u_{\mathbf{n}, \mathbf{m}^{\prime}}^{\ell} s_{\mathbf{n}}^{\ell}$. Notice that we can write $\operatorname{diag}\left[\mathbf{B}_{1}^{k} U^{k}\right] Q^{\ell}=\tilde{\mathbf{B}}_{1}^{k}\left(Q^{\ell}\right) U^{k}$ and $\operatorname{diag}\left[\mathbf{B}_{2}^{\ell} U^{\ell}\right] S^{\ell}=\tilde{\mathbf{B}}_{2}^{\ell}\left(S^{\ell}\right) U^{\ell}$, where the matrices $\tilde{\mathbf{B}}_{1}^{k}\left(Q^{\ell}\right)$ and $\tilde{\mathbf{B}}_{2}^{\ell}\left(S^{\ell}\right)$ have negative entries, since $q$ and $s$ are both nonnegative. Direct computation in terms of the coefficients shows that

$$
\begin{aligned}
& \left\|\operatorname{diag}\left[\mathbf{B}_{1}^{k} U^{k}\right] Q^{\ell}\right\|_{\infty} \leq \delta t^{2} \tilde{b}_{1}(T, \lambda, V)\left\|U^{k}\right\|_{\infty}, \\
& \left\|\operatorname{diag}\left[\mathbf{B}_{2}^{\ell} U^{\ell}\right] S^{\ell}\right\|_{\infty} \leq \delta t \tilde{b}_{2}(T, \lambda)\left\|U^{\ell}\right\|_{\infty},
\end{aligned}
$$

where $\tilde{b}_{1}(T, \lambda, V)=\Gamma\|q\|_{L_{\mathbf{x}, \mathbf{v}, t}^{\infty}} e^{\lambda T} 4 V^{2} \tilde{a}$ and $\tilde{b}_{2}(T, \lambda) \sim \chi\|s\|_{L_{\mathbf{x}, t}^{\infty}} e^{\lambda T} \int w(\mathbf{v}) d \mathbf{v}$, recalling that $M \delta v=2 V$. 
Let us consider now the nonlinear terms in (49). The elements of $\operatorname{diag}\left[D_{1}^{\ell}\left(C^{\ell}\right)-\right.$ $\left.D_{1}^{\ell}\left(S^{\ell}\right)\right] Q^{\ell}$ are $\delta t\left[\alpha\left(e^{\nu t_{\ell}} c_{\mathbf{n}}^{\ell}\right)-\alpha\left(e^{\nu t_{\ell}} s_{\mathbf{n}}^{\ell}\right)\right] \delta_{\mathbf{v}^{0}}\left(\mathbf{v}_{\mathbf{m}}\right) q_{\mathbf{n}, \mathbf{m}}^{\ell}$. The differences can be expressed in terms of $R^{\ell}$ using the mean value theorem to write $\operatorname{diag}\left[D_{1}^{\ell}\left(C^{\ell}\right)-\right.$ $\left.D_{1}^{\ell}\left(S^{\ell}\right)\right] Q^{\ell}=\mathbf{B}_{3}^{\ell}\left(Q^{\ell}\right) R^{\ell}$. We find

$$
\left|\alpha\left(e^{\nu t_{\ell}} c_{\mathbf{n}}^{\ell}\right)-\alpha\left(e^{\nu t_{\ell}} s_{\mathbf{n}}^{\ell}\right)\right|=e^{\nu t_{k}}\left|\alpha^{\prime}\left(\eta_{3} e^{\nu t_{k}}\right)\right|\left|r_{\mathbf{n}}^{\ell}\right| .
$$

If we set $\tilde{b}_{3}(T, \nu)=\frac{e^{\nu T}}{\pi \sigma_{v}^{2}}\left\|\alpha^{\prime}\right\|_{\infty}\|q\|_{L_{\mathbf{x}, \mathbf{v}, t}^{\infty}}$, we obtain the estimate

$$
\left\|\operatorname{diag}\left[D_{1}^{\ell}\left(C^{\ell}\right)-D_{1}^{\ell}\left(S^{\ell}\right)\right] Q^{\ell}\right\|_{\infty} \leq \delta t \tilde{b}_{3}(T, \nu)\left\|R^{\ell}\right\|_{\infty} .
$$

Finally, the elements of the remaining term $\left[\mathbf{D}_{2}^{\ell}\left(C^{\ell}\right)-\mathbf{D}_{2}^{\ell}\left(S^{\ell}\right)\right] Q^{\ell}$ are sums of $\beta \frac{\delta t}{\delta v}\left[F_{1}^{ \pm}\left(c_{\mathbf{n}}^{\ell}\right)-F_{1}^{ \pm}\left(s_{\mathbf{n}}^{\ell}\right)\right]\left(q_{\mathbf{n}, m_{1} \mp 1, m_{2}}^{\ell}-q_{\mathbf{n}, \mathbf{m}}^{\ell}\right)$ and $\beta \frac{\delta t}{\delta v}\left[F_{2}^{ \pm}\left(c_{\mathbf{n}}^{\ell}\right)-F_{2}^{ \pm}\left(s_{\mathbf{n}}^{\ell}\right)\right]\left(q_{\mathbf{n}, m_{1}, m_{2} \mp 1}^{\ell}-\right.$ $\left.q_{\mathbf{n}, \mathbf{m}}^{\ell}\right)$. By the mean value theorem

$$
\left|F_{1}^{+}\left(e^{\nu t_{\ell}} c_{\mathbf{n}}^{\ell}\right)-F_{1}^{+}\left(e^{\nu t_{\ell}} s_{\mathbf{n}}^{\ell}\right)\right|=\eta_{4}\left|F_{1}\left(e^{\nu t_{\ell}} c_{\mathbf{n}}^{\ell}\right)-F_{1}\left(e^{\nu t_{\ell}} s_{\mathbf{n}}^{\ell}\right)\right|=\eta_{4} e^{\nu t_{\ell}}\left|F^{\prime}\left(e^{\nu t_{\ell}} \eta_{5}\right)\right|\left|r_{\mathbf{n}}^{\ell}\right|,
$$

with $\eta_{4} \in[0,1]$. Similar arguments apply to the negative part, since $x^{-}=(-x)^{+}$, and to the component $F_{2}$. Thus

$$
\left\|\left[\mathbf{D}_{2}^{\ell}\left(C^{\ell}\right)-\mathbf{D}_{1}^{\ell}\left(S^{\ell}\right)\right] Q^{\ell}\right\|_{\infty} \leq \delta t \tilde{b}_{4}(T, \nu)\left\|R^{\ell}\right\|_{\infty},
$$

where $b_{4}(T, \nu) \sim e^{\nu T}\left\|F^{\prime}\right\|_{\infty}\left\|\nabla_{\mathbf{v}} q\right\|_{L_{\mathbf{x}, \mathbf{v}, t}^{\infty}}$.

Now, let us set $\mathcal{A}\left(U^{\ell}, R^{\ell}\right)=\operatorname{diag}\left[\sum_{k=0}^{\ell} \mathbf{B}_{1}^{k} U^{k}+\left[D_{1}^{\ell}\left(C^{\ell}\right)-D_{1}^{\ell}\left(S^{\ell}\right)\right]\right] Q^{\ell}+$ $\left[\mathbf{D}_{2}^{\ell}\left(C^{\ell}\right)-\mathbf{D}_{2}^{\ell}\left(S^{\ell}\right)\right] Q^{\ell}$ and $K^{\ell}=\operatorname{Max}\left\{\left\|R^{\ell}\right\|_{\infty},\left\|U^{\ell}\right\|_{\infty}\right\}$. Combining (51) and (52)-(54), we find

$$
\begin{aligned}
& \left\|\tilde{\mathbf{A}}_{1}^{\ell} U^{\ell}+\mathcal{A}\left(U^{\ell}, R^{\ell}\right)\right\|_{\infty} \leq\left(1+\gamma^{\prime} \delta t\right) \operatorname{Max}_{k \leq \ell} K^{k}, \\
& \left\|\tilde{\mathbf{A}}_{2}^{\ell} R^{\ell}+\operatorname{diag}\left[\mathbf{B}_{2}^{\ell} U^{\ell}\right] S^{\ell}\right\|_{\infty} \leq\left(1+\gamma^{\prime} \delta t\right) K^{\ell},
\end{aligned}
$$

for a certain value $\gamma^{\prime}$, since $\ell \delta t \leq \mathcal{L} \delta t \leq T$. Inserting estimate (55) in (49)-(50) we find

$$
\begin{aligned}
& \left\|U^{\ell+1}\right\|_{\infty} \leq\left(1+\gamma^{\prime} \delta t\right) \operatorname{Max}_{k \in\{0, \ldots, \ell\}} K^{k}+W^{\ell}, \\
& \left\|R^{\ell+1}\right\|_{\infty} \leq\left(1+\gamma^{\prime} \delta t\right) K^{\ell}+W^{\ell},
\end{aligned}
$$

where $W^{\ell}=\operatorname{Max}\left\{\left\|Z^{\ell}\right\|_{\infty},\left\|Y^{\ell}\right\|_{\infty}\right\}$. When we take $\mathbf{F}$ to be a given smooth field, the contributions from (54) vanish, but this final estimate holds. Therefore, in both cases (given $\mathbf{F}$ or coupled $\mathbf{F}(c)$ ) we have

$$
\operatorname{Max}_{k \in\{0, \ldots, \ell+1\}} K^{k} \leq \tilde{\gamma}(\delta t) \operatorname{Max}_{k \in\{0, \ldots, \ell\}} K^{k}+\operatorname{Max}_{k \in\{0, \ldots, \ell\}} W^{k},
$$

where $\tilde{\gamma}(\delta t)=1+\gamma^{\prime} \delta t$. Let us assume $\gamma^{\prime}>0$. Since $K^{0}=0$, this inequality implies:

$$
\operatorname{Max}_{k \in\{1, \ldots, \ell+1\}} K^{k} \leq \sum_{j=0}^{\ell} \tilde{\gamma}(\delta t)^{j} \operatorname{Max}_{k \in\{1, \ldots, \ell\}} W^{k}=O(\delta t+\delta x+\delta v),
$$


since $\operatorname{Max}_{k \in\{0, \ldots, \ell\}} W^{k}=\delta t O(\delta t+\delta x+\delta v), \tilde{\gamma}(\delta t)^{j} \leq\left(1+\gamma^{\prime} \delta t\right)^{\ell} \leq e^{\gamma^{\prime} \ell \delta t} \leq e^{\gamma^{\prime} T}$ and $\delta t(\ell+1) \leq T$. If $\gamma^{\prime}<0$ then $\delta t \sum_{j=0}^{\ell} \tilde{\gamma}(\delta t)^{j} \leq\left|\gamma^{\prime}\right|^{-1}$. Therefore, the solutions of the discretized problem converge to the solutions of the original problem in our computational region, with an error of order one.

Some remarks are in order now. First, notice that $(1-\gamma \delta t)<1$ in $(51)$ is not really necessary, $(1-\gamma \delta t) \leq 1$ is enough. Only the hypothesis (H0) on $\lambda$ is really needed to ensure positivity of the solutions and to guarantee the additional bounds on the required norms. Thus, we can set $\nu=0$.

In principle, Theorem 2.4. could be extended to handle nonlocal boundary conditions of the form (30)-(31). As pointed out in Proposition 2.1, the discretized boundary operators $S_{0}^{d}(p)$ and $S_{L}^{d}(p)$ preserve positivity. Moreover, they are bounded in $\ell^{\infty}$. Indeed, the boundary values are bounded by

$$
\begin{aligned}
0 \leq p_{0, n_{2}, \mathbf{m}}^{\ell+1} & =S_{0}^{d}\left(p^{\ell}\right) \leq \frac{2}{\sqrt{\pi}} e^{-\left|\mathbf{v}_{\mathbf{m}}-\mathbf{v}^{0}\right|^{2}}\left[\frac{A v_{1}^{0}}{\pi \sigma_{v}^{2}}+4 \tilde{a} V^{3}\right]\left\|p_{\mathbf{n}, \mathbf{m}}^{\ell}\right\|_{\infty}, \\
0 & \leq p_{N_{1}, n_{2}, \mathbf{m}}^{\ell+1}=S_{L}^{d}\left(p^{\ell}\right) \leq \frac{2}{\pi} e^{-\left|\mathbf{v}_{\mathbf{m}}-\mathbf{v}^{0}\right|^{2}} 4 \tilde{a} V^{2}\left\|p_{\mathbf{n}, \mathbf{m}}^{\ell}\right\|_{\infty},
\end{aligned}
$$

since $M \delta v=2 V$. The matrix $\mathbf{A}_{1}$ in (26) representing the discretization of the linear differential operators should be corrected now to include these linear nonlocal operators. Additional hypotheses on the parameters defining the boundary operators are needed to guarantee that the updated matrix $\mathbf{A}_{1}$ would still satisfy $\left\|\mathbf{A}_{1}\right\|_{\infty} \leq 1$. Under such conditions, the proof of Theorem 2.4 could be adapted to provide convergence in this case too. However, notice that the simulations performed in Section 3 start from initial densities that are exponentially small at all boundaries, and the boundary conditions remain basically zero during the soliton migration. This is the main context we have in mind in this paper.

Extending Theorem 2.4. to force fields $\mathbf{F}(C)$ involving gradients and discrete gradients of $C$ as in (4) and (29) encounters the following technical difficulty. To obtain (54), we should estimate

$$
\begin{array}{r}
{\left[F_{1}\left(e^{\nu t_{\ell}} c_{\mathbf{n}}^{\ell}\right)^{+}-F_{1}\left(e^{\nu t_{\ell}} s_{\mathbf{n}}^{\ell}\right)^{+}\right]=\left[h\left(e^{\nu t_{\ell}} c_{\mathbf{n}}^{\ell}\right)-h\left(e^{\nu t \ell} s_{\mathbf{n}}^{\ell}\right)\right] e^{\nu t_{\ell}}\left(\frac{s_{n_{1}+1, n_{2}}^{\ell}-s_{n_{1}, n_{2}}^{\ell}}{\delta x}\right)^{+}} \\
+h\left(e^{\nu t_{\ell}} c_{\mathbf{n}}^{\ell}\right) e^{\nu t_{\ell}}\left[\left(\frac{c_{n_{1}+1, n_{2}}^{\ell}-c_{n_{1}, n_{2}}^{\ell}}{\delta x}\right)^{+}-\left(\frac{s_{n_{1}+1, n_{2}}^{\ell}-s_{n_{1}, n_{2}}^{\ell}}{\delta x}\right)^{+}\right]= \\
h^{\prime}\left(e^{\nu t_{\ell}} \xi_{1}\right) e^{\nu t_{\ell}} r_{\mathbf{n}}^{\ell}\left(\frac{s_{n_{1}+1, n_{2}}^{\ell}-s_{n_{1}, n_{2}}^{\ell}}{\delta x}\right)^{+}+\xi_{2} h\left(e^{\nu t_{\ell}} c_{\mathbf{n}}^{\ell}\right) e^{\nu t_{\ell}}\left(\frac{r_{n_{1}+1, n_{2}}^{\ell}-r_{n_{1}, n_{2}}^{\ell}}{\delta x}\right) .
\end{array}
$$

The first term is bounded by $\delta_{1} q_{1} \Gamma_{1} e^{2 \nu T}\|\nabla s\|_{L_{\mathbf{x} t}^{\infty}}\left|r_{\mathbf{n}}\right|$ and can be handled as in the proof. The second term, however, involves a 'discrete derivative'. We would need to estimate $d r_{\mathbf{n}}^{\ell}=\frac{r_{n_{1}+1, n_{2}}^{\ell}-r_{n_{1}, n_{2}}^{\ell}}{\delta x}$ in terms of $\left\|R^{\ell}\right\|_{\infty}$ and $\left\|U^{\ell}\right\|_{\infty}$. A continuous version of this difficulty is encountered when proving existence of solutions for the continuous model as limits of solutions of linearized problems with nonlinear coefficients frozen in the previous iterate at each iteration in Refs. [13] and [14]. The gradients of the concentrations are estimated there 
thanks to integral expressions in terms of semigroups of the solutions of heat equations $c_{t}-\kappa \Delta c=h$ and to decay properties of the heat kernels generating such semigroups in the whole space and the bounded domains of choice. More precisely, we have

$$
c(t)=\int_{0}^{t} S(t-s) h(s) d s, \quad\|\nabla(S(t) h)\|_{\infty} \leq \mu t^{-1 / 2}\|h\|_{\infty}, \mu>0 .
$$

This yields $\|\nabla c\|_{\infty} \leq t^{1 / 2}\|h\|_{\infty}$. Such estimates of heat semigroups are not known for all domains, but they have been proved for annular regions and slabs, for instance. From (27), (34), (48) and (50), we see that

$$
r_{\mathbf{n}}^{\ell+1}=\left[1-4 \frac{\delta t}{\delta x^{2}} \kappa\right] \tilde{r}_{\mathbf{n}}^{\ell}+\frac{\delta t}{\delta x^{2}} \kappa\left[\tilde{r}_{n_{1}+1, n_{2}}^{\ell}+\tilde{r}_{n_{1}-1, n_{2}}^{\ell}+\tilde{r}_{n_{1}, n_{2}+1}^{\ell}+\tilde{r}_{n_{1}, n_{2}-1}^{\ell}\right]+\delta t h_{\mathbf{n}}^{\ell},
$$

where $h_{\mathbf{n}}^{\ell}=-\chi j^{d}\left(p_{\mathbf{n}, \mathbf{m}}^{\ell}\right) r_{\mathbf{n}}^{\ell}-\chi j^{d}\left(u_{\mathbf{n}, \mathbf{m}}^{\ell}\right) s_{\mathbf{n}}^{\ell}-y_{\mathbf{n}}^{\ell}$, with zero initial and boundary data. In matrix form, we would have

$$
R^{\ell+1}=\left(\mathcal{I}+\frac{\delta t}{\delta x^{2}} \kappa \mathcal{N}\right) R^{\ell}+\delta t H^{\ell}=\delta t \sum_{j=0}^{\ell}\left(\mathcal{I}+\delta t \frac{\delta t}{\delta x^{2}} \kappa \mathcal{N}\right)^{\ell-j} H^{j} .
$$

Denoting by $d R^{\ell}$ the vector whose components are 'discrete derivatives' of the components of $R^{\ell}$, a bound of the form $\left\|d R^{\ell}\right\|_{\infty} \leq \mu \sum_{j \leq \ell}\left\|H^{j}\right\|_{\infty}$ is available when the vectors $H^{j}$ are equal and constant (we can add the matrices). However, it is not true in general, as it can be seen at the first step already: $r_{\mathbf{n}}^{1}=\delta t h_{\mathbf{n}}^{1}$ and we cannot obtain information on $\frac{r_{n_{1}+1, n_{2}}^{1}-r_{n_{1}, n_{2}}^{1}}{\delta x}$ without information on $\frac{h_{n_{1}+1, n_{2}}^{1}-h_{n_{1}, n_{2}}^{1}}{\delta x}$, which would require unavailable information on $\frac{u_{n_{1}+1, n_{2}, \mathbf{n}}^{1}-u_{n_{1}, n_{2}, \mathbf{m}}^{1}}{\delta x}$. The situation is repeated at the next time levels. Whether other discretizations of heat problems can keep positivity and provide bounds on discrete gradients similar to the continuous ones is a matter of study.

To conclude, a fully convergent scheme for forces (4) would be obtained in two steps. First, we approximate the continuous problem by a sequence of continuous problems with frozen force fields, as in the existence proofs $[13,14]$. Then, we approximate numerically the solution of each of these problems by the present scheme with given force field. However, it is computationally more efficient to implement directly (26)-(27) with (28) and (29). The next sections show that this scheme is quite reliable to track smooth solitons.

\section{Simulation of soliton-like behavior}

In this section, we present numerical solutions for appropriate values of the parameters as listed in Table 1 , setting in addition $a=1 / 0.3$ and $c_{L}(t)=1.1$ in the boundary condition (8). The slab geometry of the advancing angiogenic network is depicted in Figure 1.

Figures 2-4 illustrate the evolution of a soliton-like numerical solution generated applying the scheme (26)-(27) with (28) and (29) to the initial data:

$$
\begin{array}{r}
p\left(x_{1}, x_{2}, v_{1}, v_{2}, 0\right)=\frac{2}{\pi^{2}} \frac{1}{0.0048} e^{-\left(\frac{x_{1}}{0.06}\right)^{2}} \sum_{j=1}^{20} e^{-\left(\frac{x_{2}-x_{2}^{j}}{0.08}\right)^{2}} e^{-\left[\left(v_{1}-v_{1}^{0}\right)^{2}+\left(v_{2}-v_{2}^{0}\right)^{2}\right]}, \\
C\left(x_{1}, x_{2}, 0\right)=1.1 e^{-\left[\left(\frac{x_{1}-1}{1.5}\right)^{2}+\left(\frac{x_{2}}{0.3}\right)^{2}\right]},
\end{array}
$$



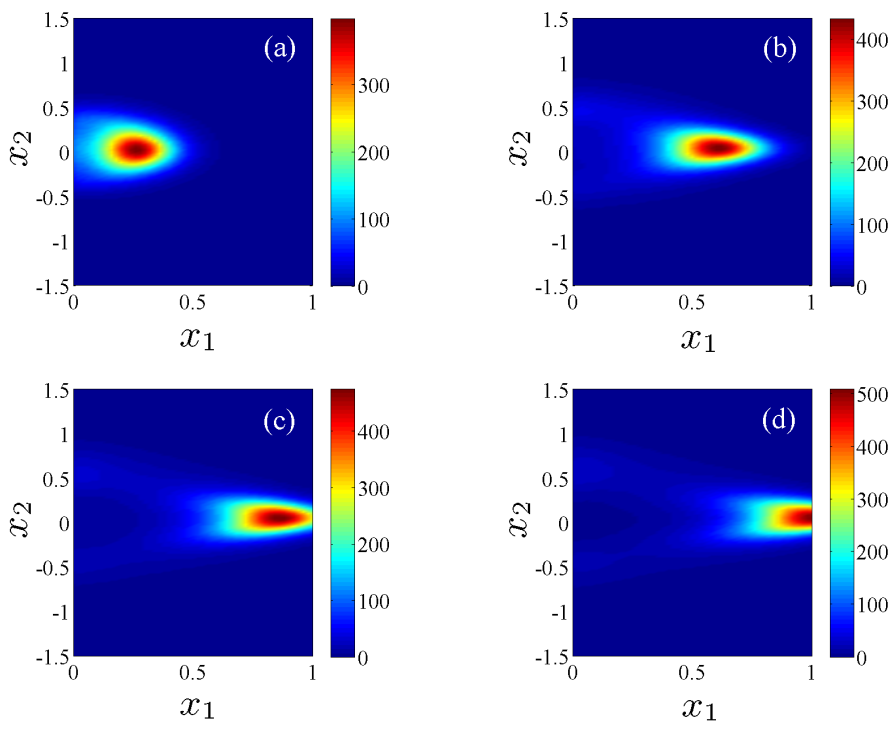

Figure 2: Density plot of the marginal vessel tip density $\rho(\mathbf{x}, t)$ at (a) 12 hours, (b) 24 hours, (c) 32 hours, (d) 36 hours.

where the vessel tip locations $x_{2}^{j}=-0.3+(j-1) \frac{0.6}{19}, j=1,2, \ldots, 20$, are 20 equispaced values in $[-0.3,0.3], v_{1}^{0}=\cos (\pi / 10)$ and $v_{2}^{0}=\sin (\pi / 10)$. The computational velocity domain is $\Omega_{\mathbf{v}}=[-4,4]^{2}$ and the spatial domain $\Omega_{\mathbf{x}}=$ $[0,1] \times[-1.5,1.5]$. The steps are $\delta x=0.02, \delta v=0.08$ and $\delta t=2.2906 \times 10^{-4}$. We discretize the boundary conditions (9)-(10) for $p$ and (8) for $C$ at $x_{1}=0,1$ using (30)-(31) and (32). Since we introduce artificial boundaries at $x_{2}= \pm 1.5$ we need additional boundary conditions. We impose zero Dirichlet boundary conditions for $C$ at $x_{2}= \pm 1.5$, as well as zero boundary conditions of the form (13) for $p$. On $\partial \Omega_{\mathbf{v}}, p$ vanishes.

Figure 2 shows the evolution of the marginal tip density (5) at four different times as the angiogenic network moves towards the tumor. Figures 3 and 4 depict the tumor angiogenic factor (TAF) concentration and the flux at the same times. We observe that the active vessel tips evolve as a patch as they consume TAF in their advance. The tip density profile forms a soliton-like pulse, as shown in Figure 5. The pulse forms at an initial stage and then moves keeping its shape but changing its height and size.

This numerically observed soliton can be described asymptotically as explained in Appendix A. In the limit as $\beta \rightarrow \infty$, the marginal density $\rho(\mathbf{x}, t)$ 

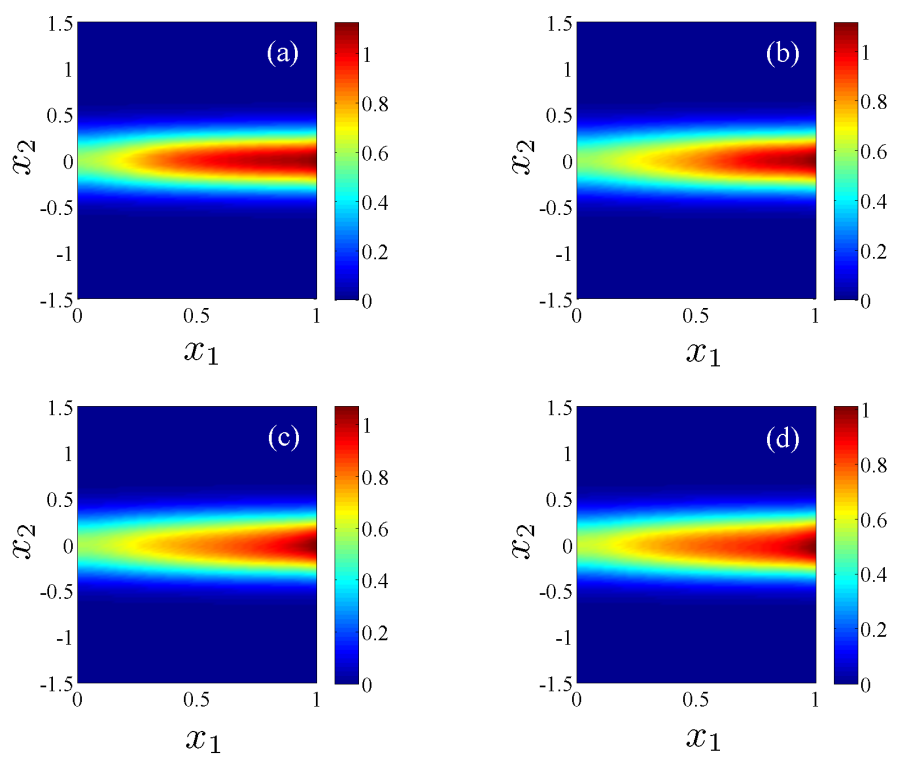

Figure 3: Density plot of the TAF $C(\mathbf{x}, t)$ at the same times as in Figure 2.
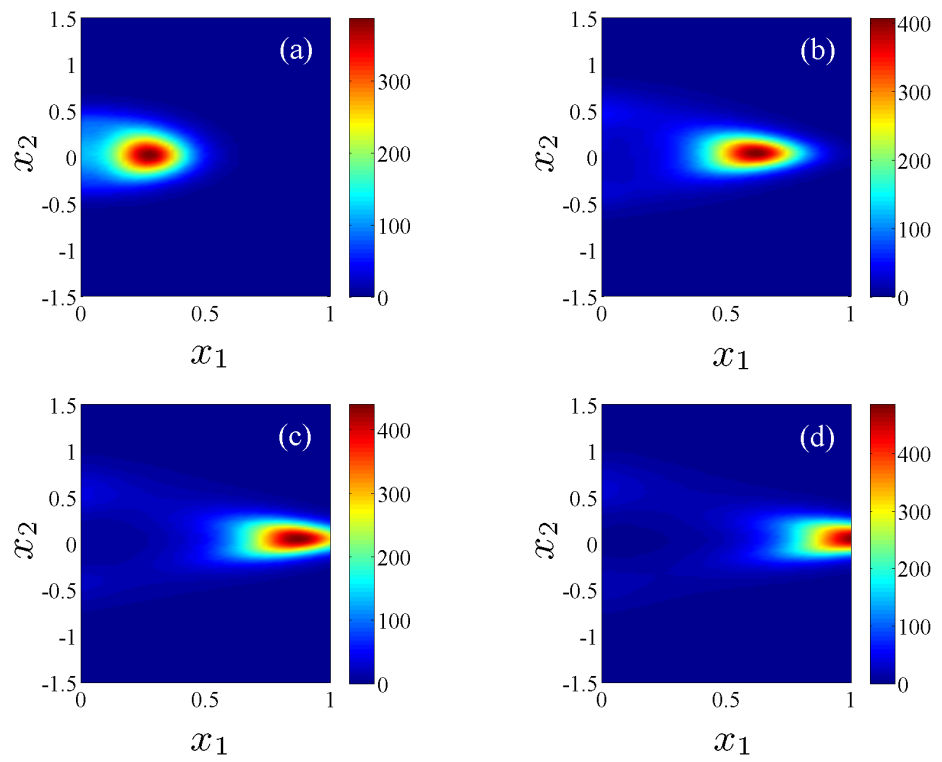

Figure 4: Density plot of the flux $j(\mathbf{x}, t)$ at the same times as in Figure 2. 

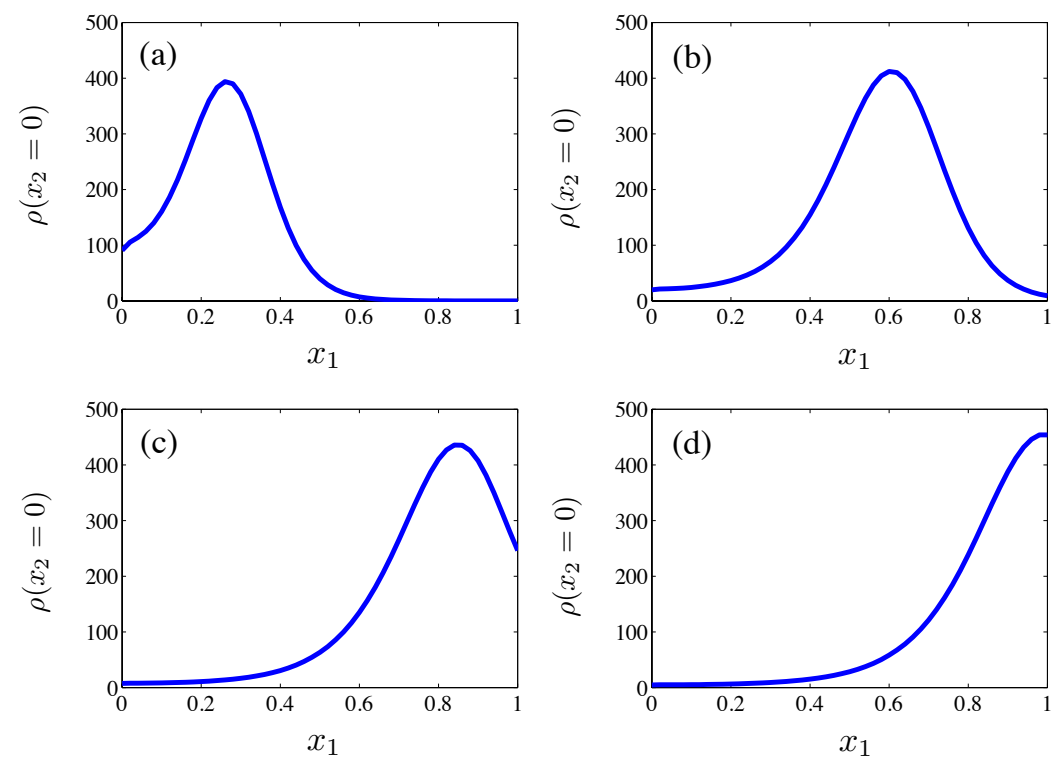

Figure 5: Profiles of the marginal tip density $\rho\left(x_{1}, 0, t\right)$ at the same times as in Figure 2.

defined in (5) satisfies the equation:

$$
\begin{array}{r}
\frac{\partial \rho}{\partial t}+\operatorname{div}_{\mathbf{x}}(\mathbf{F} \rho)-\frac{1}{2 \beta} \Delta_{\mathbf{x}} \rho=\mu \rho-\Gamma \rho \int_{0}^{t} \rho(\mathbf{x}, s) d s \\
\mu=\frac{\alpha}{\pi}\left[1+\frac{\alpha}{2 \pi \beta\left(1+\sigma_{v}^{2}\right)} \ln \left(1+\frac{1}{\sigma_{v}^{2}}\right)\right]
\end{array}
$$

To leading order, the density and the marginal density are related by

$$
p(\mathbf{x}, \mathbf{v}, t) \sim \frac{1}{\pi} e^{-\left|\mathbf{v}-\mathbf{v}^{0}\right|^{2}} \rho(\mathbf{x}, t)
$$

Inserting this in the TAF equation (2) with $j$ given by (5), we obtain

$$
\begin{aligned}
& \frac{\partial}{\partial t} C(\mathbf{x}, t)=\kappa \Delta_{\mathbf{x}} C(\mathbf{x}, t)-\chi_{1} C(\mathbf{x}, t) \rho(\mathbf{x}, t) \\
& \chi_{1}=\frac{\chi}{\pi} \int_{0}^{\infty} \int_{-\pi}^{\pi} \frac{\sqrt{1+V^{2}+2 V \cos \varphi}}{1+e^{\left(V^{2}-\eta\right) / \epsilon}} e^{-V^{2}} V d V d \varphi
\end{aligned}
$$

Numerical simulations show that the density of active vessel tips approaches a moving two-dimensional lump, which we call the 'angiton', after an initial formation stage. The longitudinal profile of the angiton is a soliton-like wave 
that solves (58) for constant $\mu, \mathbf{F} \cdot \hat{\mathbf{n}}=F_{1}$ and $1 / \beta=0$, namely,

$$
\begin{aligned}
& \rho_{s}\left(x_{1} ; K, c, X\right)=\frac{\left(2 K \Gamma+\mu^{2}\right) c}{2 \Gamma\left(c-F_{1}\right)} \operatorname{sech}^{2}\left[\frac{\sqrt{2 K \Gamma+\mu^{2}}}{2\left(c-F_{1}\right)}\left(x_{1}-X\right)\right], \\
& \dot{X}=\frac{d X}{d t}=c
\end{aligned}
$$

where $X(t)$ denotes the position of the maximum and $c$ the propagation speed. Here the parameters $K$ and $c$, called collective coordinates, satisfy two ordinary differential equations that are obtained assuming that the TAF varies slowly in space and time [5]. For the parameters used in the numerical simulations, these equations are

$$
\begin{aligned}
& \dot{K}=\frac{\left(2 K \Gamma+\bar{\mu}^{2}\right)^{2}}{4 \Gamma \beta\left(c-\overline{F_{1}}\right)^{2}} \frac{\frac{4 \pi^{2}}{75}+\frac{1}{5}+\left(\frac{2 \overline{F_{1}}}{5 c}-\frac{2 \pi^{2}}{75}-\frac{9}{10}\right) \frac{\overline{F_{1}}}{c}}{\left(1-\frac{4 \pi^{2}}{15}\right)\left(1-\overline{F_{1}} \frac{\overline{2}}{2 c}\right)^{2}} \\
& -\frac{2 K \Gamma+\bar{\mu}^{2}}{2 \Gamma c\left(1-\frac{\overline{F_{1}}}{2 c}\right)}\left(c \overline{\operatorname{div}_{\mathbf{x}} \mathbf{F}}+\overline{\mathbf{F} \cdot \nabla_{\mathbf{x}} F_{1}}-\frac{\overline{\Delta_{\mathbf{x}} F_{1}}}{2 \beta}\right), \\
& \dot{c}=-\frac{7\left(2 K \Gamma+\bar{\mu}^{2}\right)}{20 \beta\left(c-\overline{F_{1}}\right)} \frac{1-\frac{4 \pi^{2}}{105}}{\left(1-\frac{4 \pi^{2}}{15}\right)\left(1-\frac{\overline{F_{1}}}{2 c}\right)}+\frac{\overline{\mathbf{F} \cdot \nabla_{\mathbf{x}} F_{1}}-\left(c-\overline{F_{1}}\right) \overline{\nabla_{\mathbf{x}} \cdot \mathbf{F}}-\frac{\overline{\Delta_{\mathbf{x}} F_{1}}}{2 \beta}}{2-\frac{\overline{F_{1}}}{c}},
\end{aligned}
$$

in which the functions of $C\left(x_{1}, x_{2}, t\right)$ have been averaged in $x_{1}$ over a subinterval $\mathcal{I} \subset(0, L):$

$$
\overline{f\left(x_{1}, x_{2}\right)}=\frac{1}{|\mathcal{I}|} \int_{\mathcal{I}} f\left(x_{1}, 0\right) d x_{1}
$$

and we have set $x_{2}=0$.

Figure 6 compares the profiles of the marginal tip density after 24 hours calculated by solving (1)-(2) in two different ways: (a) with the flux (5) we consider here (solid blue line) and (b) with flux $|\mathbf{j}(\mathbf{x}, t)|$, with $\mathbf{j}$ given by (7) as in [42] (dashed blue line). The anastomosis coefficients $\Gamma$ have been fitted so that the solutions of the corresponding deterministic equations agree with averages of the stochastic process explained in the next section. Except for the different values of $\Gamma$, the different definition of the flux in (2) does not affect much the results because the TAF concentration varies slowly in time for the parameters used in the numerical simulations. In addition, we have fit the asymptotic shape of the soliton, given by (63). Figure 6 shows that they agree well. More detailed comparisons between the motion of the soliton and the numerical solution of the full kinetic equations for exponentially decaying data without velocity cut-offs and the flux $\left|\int \mathbf{v} p(\mathbf{x}, \mathbf{v}, t) d \mathbf{v}\right|$ can be found in [5].

\section{Comparison with the stochastic model and velocity cut-offs}

The stochastic model of blood vessel growth [3, 42] consists of a system of Langevin equations for the extension of vessel tips, a tip branching process and 


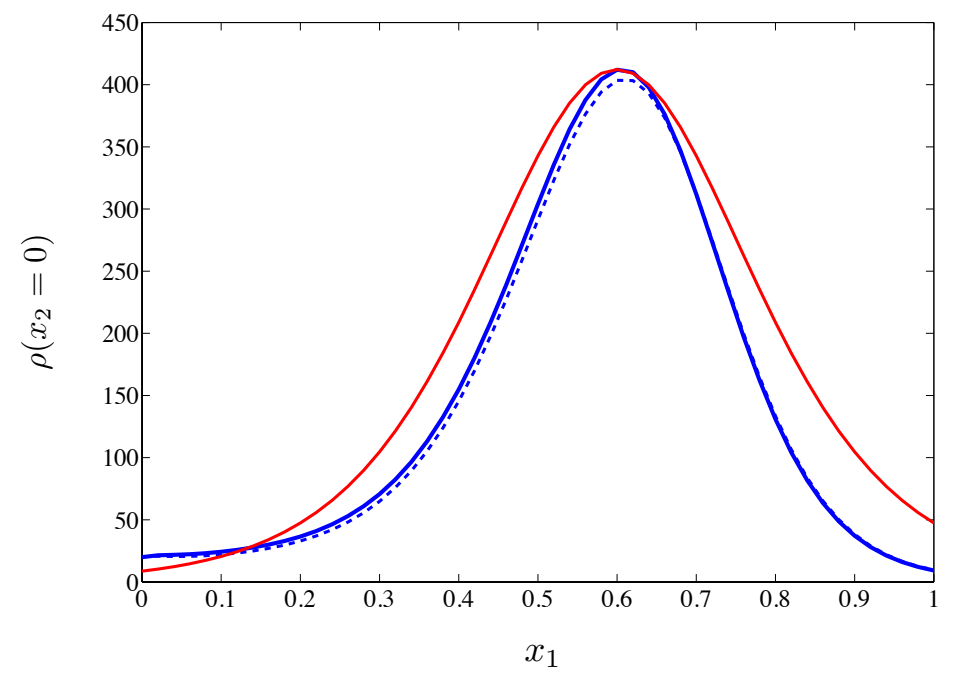

Figure 6: Profiles of the marginal tip density $\rho\left(x_{1}, 0, t\right)$ at $24 \mathrm{hr}$ : numerical solution of the deterministic equations (1)-(2) with flux (5) and $\Gamma=0.135$ (solid blue line); numerical solution with flux $\left|\int \mathbf{v} p(\mathbf{x}, \mathbf{v}, t) d \mathbf{v}\right|$ and $\Gamma=0.145$ (dashed blue line); soliton solution fit to the numerical solution with flux (5) (red line).

tip annihilation (anastomosis) when they merge with existing vessels. A tip $i$ is born at a random time $T^{i}$ from a moving tip (we ignore branching from mature vessels) and disappears at a later random time $\Theta^{i}$, either by reaching the tumor or by anastomosis. We recall next how these processes are accounted for.

Branching. At time $T^{i}$, the velocity of the newly created tip $i$ is selected out of a normal distribution (6) and, for a given realization $\omega$ of the stochastic process, the number of active tips at time $t$ is $N(t, \omega)$. The probability that a tip branches from one of the existing ones during an infinitesimal time interval $(t, t+d t]$ is taken proportional to $\sum_{i=1}^{N(t, \omega)} \alpha\left(C\left(\mathbf{X}^{i}(t), t\right)\right) d t$, where $\alpha(C)$ is given by (4). The change per unit time of the number of tips in boxes $d \mathbf{x}$ and $d \mathbf{v}$ about $\mathbf{x}$ and $\mathbf{v}$ is

$$
\begin{aligned}
& \sum_{i=1}^{N(t, \omega)} \alpha\left(C\left(\mathbf{X}^{i}(t), t\right)\right) \delta_{\sigma_{v}}\left(\mathbf{v}^{i}(t)-\mathbf{v}^{0}\right)=\int_{d \mathbf{x}} \int_{d \mathbf{v}} \alpha(C(\mathbf{x}, t)) \\
& \times \delta_{\sigma_{v}}\left(\mathbf{v}-\mathbf{v}^{0}\right) \sum_{i=1}^{N(t, \omega)} \delta_{\sigma_{x}}\left(\mathbf{x}-\mathbf{X}^{i}(t)\right) \delta_{\sigma_{v}}\left(\mathbf{v}-\mathbf{v}^{i}(t)\right) d \mathbf{x} d \mathbf{v},
\end{aligned}
$$

where $\mathbf{X}^{i}(t)$ and $\mathbf{v}^{i}(t)$ are the position and velocity of tip $i$ at time $t$, respectively. Tip extensions. The Langevin equations are

$$
\begin{aligned}
& d \mathbf{X}^{i}(t)=\mathbf{v}^{i}(t) d t, \\
& d \mathbf{v}^{i}(t)=\beta\left[-\mathbf{v}^{i}(t)+\mathbf{F}\left(C\left(\mathbf{X}^{i}(t), t\right)\right)\right] d t+\sqrt{\beta} d \mathbf{W}^{i}(t),
\end{aligned}
$$


where $\mathbf{W}^{i}(t)$ are independent identically distributed (i.i.d.) standard Brownian motions, and the positive parameter $\beta$ is a dimensionless friction coefficient. The chemotactic force is given by (4) and the TAF concentration solves

$$
\frac{\partial}{\partial t} C(\mathbf{x}, t)=\kappa \Delta_{\mathbf{x}} C(\mathbf{x}, t)-\chi C(\mathbf{x}, t) \sum_{i=1}^{N(t, \omega)}\left|\mathbf{v}^{i}(t)\right| \delta_{\sigma_{x}}\left(\mathbf{x}-\mathbf{X}^{i}(t)\right) .
$$

Here $\kappa$ (diffusivity) and $\chi$ are positive dimensionless parameters, whereas $\delta_{\sigma_{x}}(\mathbf{x}-$ $\mathbf{X}^{i}(t)$ ) is a regularized smooth delta function similar to (6) (e.g., a Gaussian) that becomes $\delta\left(\mathbf{x}-\mathbf{X}^{i}(t)\right)$ in the limit as the variances go to zero. We have eliminated the cut-off by setting $\eta \rightarrow \infty$ in (5).

Anastomosis. In two space dimensions, when an active tip meets a point of the trajectory of another tip, it stops there and ceases to be active. The same occurs when two active tips meet at the same point or when an active tip reaches the tumor.

Remark. In three space dimensions, tip cells are modelled as spheres and their trajectories are tubes. Anastomosis occurs when a tip cell touches a tube representing the trajectory of another tip cell. Instead of this hard spheres vision, the size of the blood vessel can be considered through some kernel function that has a sharp peak inside a sphere of radius twice the tip radius (cf. Ref. [10]).

The deterministic description in terms of integrodifferential equations (1)-(2) for the tip density and the TAF concentration is obtained from the stochastic description as follows. Let us define the following ensemble averages over a sufficient number $\mathcal{N}$ of replicas (realizations) $\omega$ of the stochastic process:

$$
\begin{aligned}
p_{\mathcal{N}}(\mathbf{x}, \mathbf{v}, t) & =\frac{1}{\mathcal{N}} \sum_{\omega=1}^{\mathcal{N}} \sum_{i=1}^{N(t, \omega)} \delta_{\sigma_{x}}\left(\mathbf{x}-\mathbf{X}^{i}(t, \omega)\right) \delta_{\sigma_{v}}\left(\mathbf{v}-\mathbf{v}^{i}(t, \omega)\right) \\
\rho_{\mathcal{N}}(\mathbf{x}, t) & =\frac{1}{\mathcal{N}} \sum_{\omega=1}^{\mathcal{N}} \sum_{i=1}^{N(t, \omega)} \delta_{\sigma_{x}}\left(\mathbf{x}-\mathbf{X}^{i}(t, \omega)\right) \\
j_{\mathcal{N}}(\mathbf{x}, t) & =\frac{1}{\mathcal{N}} \sum_{\omega=1}^{\mathcal{N}} \sum_{i=1}^{N(t, \omega)} \frac{\left|\mathbf{v}^{i}(t, \omega)\right|}{1+e^{\left(\left|\mathbf{v}^{i}(t, \omega)-\mathbf{v}^{0}\right|^{2}-\eta\right) / \epsilon}} \delta_{\sigma_{x}}\left(\mathbf{x}-\mathbf{X}^{i}(t, \omega)\right)
\end{aligned}
$$

As $\mathcal{N} \rightarrow \infty$, these ensemble averages tend to the tip density $p(t, \mathbf{x}, \mathbf{v})$, the marginal tip density $\rho(t, \mathbf{x})$, and the tip flux $j(t, \mathbf{x})$, respectively. The heuristic derivation of (1)-(2) from the stochastic process can be found in [42] (except for a slightly different definition of the tip flux, see (7)).

To solve the Ito stochastic differential equations of the model, (69), we have used a standard stochastic Euler-Maruyama method [24] with time step $d t=$ 0.003. At each time step $d t$ and for each tip $i$, we extract a random number $U$ with equal probability between 0 and 0.4 . A new tip branches out from $i$ at $\mathbf{x}=\mathbf{X}^{i}(t)$ only if $U<\alpha\left(C\left(\mathbf{X}^{i}(t), t\right)\right) d t$. Its initial position is $\mathbf{x}$ and its initial 


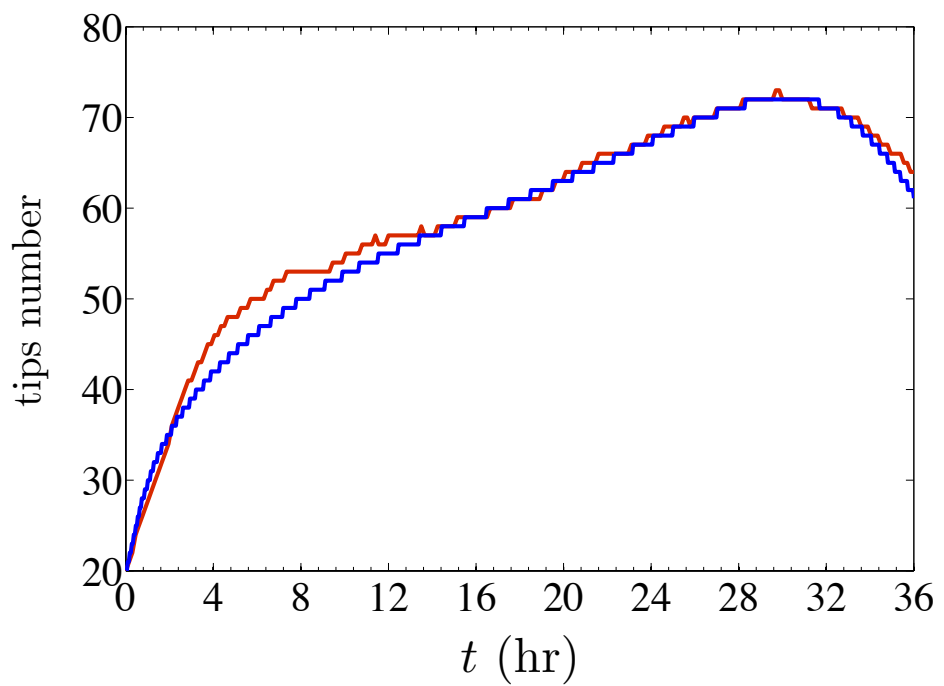

Figure 7: Evolution of the total number of vessel tips given by the integer part of $\int \rho(\mathbf{x}, t) d \mathbf{x}$ in the kinetic model (blue) as compared to that calculated from the ensemble average over 400 replicas of the stochastic process (red).

velocity is selected out of a normal distribution with mean $\mathbf{v}^{0}$ and variance $\sigma_{v}^{2}$. We have compared the numerical solution of the kinetic model with the solutions of the stochastic model through magnitudes that can be evaluated using both, such as the total number of active tips. For the stochastic process, we calculate the total number of active tips as the number of active tips at time $t$ averaged over a sufficiently large number of replicas (realizations) of the stochastic process. Typically, ensemble averages do not vary appreciable for $\mathcal{N} \geq 400$ and, therefore, we usually average over 400 replicas. As observed in Figure 7, the total number of active tips keeps increasing until the pulse front reaches the tumor. Then it decreases as tips that reach the tumor become inactive.

The model we study in this paper introduces a velocity cut-off through the flux $j$. The original model in [42] considers equations (1) and (2) with a different flux: $j(\mathbf{x}, t)=\left|\int \mathbf{v} p(\mathbf{x}, \mathbf{v}, t) d \mathbf{v}\right|$. The boundary conditions were also (8), (9), (10), (11) and (12), except for the different definition of the flux $j$. However, global in time existence results $[13,14]$ require the introduction of velocity cutoff in the flux $j$, similar to the one we impose in (5), for general integrable data with algebraic velocity decay. Comparing the simulations in [42] with data decaying exponentially fast, and those here for the same data using weighted $j$ given by (5), we see similar results.

In Ref. [42], the value $\Gamma=0.145$ produced the best fit of the total number of active tips to that determined by ensemble average over 400 replicas of the stochastic process. With the flux given by (5), the best fit with ensemble averages of the stochastic process is obtained for $\Gamma=0.135$. We observe 


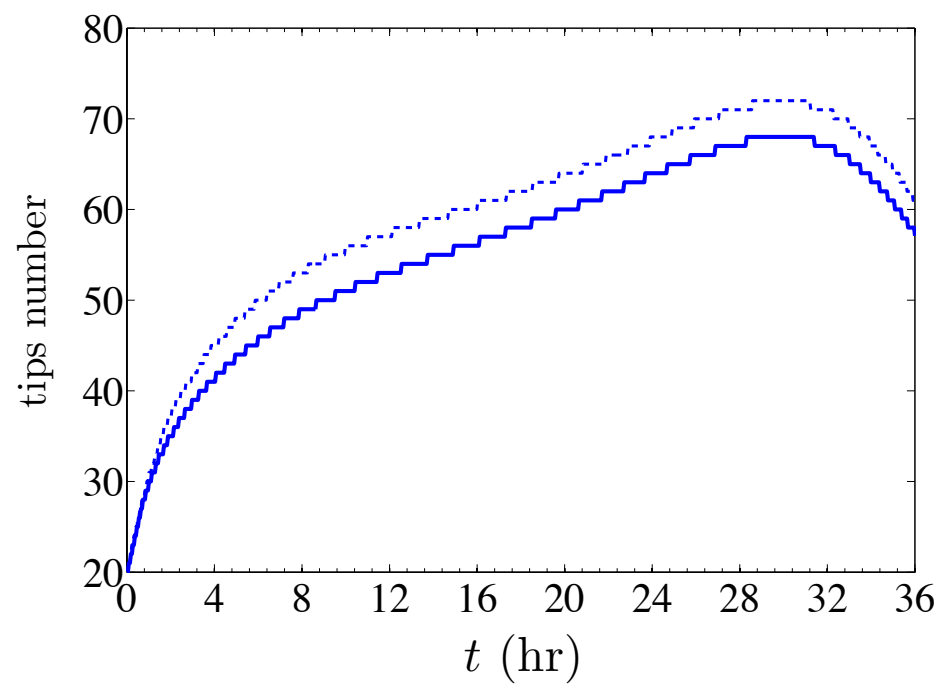

Figure 8: Evolution of the total number of vessel tips given by the integer part of $\int \rho(\mathbf{x}, t) d \mathbf{x}$ for $j(\mathbf{x}, t)$ given by (5) (solid line) as compared to that calculated for $j(\mathbf{x}, t)=\left|\int \mathbf{v} p(\mathbf{x}, \mathbf{v}, t) d \mathbf{v}\right|$ and $\Gamma=0.145$ (dashed line) [42].

that the difference in the form of $j(\mathbf{x}, t)$ produces a different mean value of the total number of active tips, as shown in Figure 8. The flux as given by (5), which contains a cut-off in the velocities, results in a smaller number of active tips. This difference is compensated by using a smaller value of the anastomosis coefficient $\Gamma$. Other than this, the numerical solution of the model equations produces quite similar results because branching yields new tips with velocities close to $\mathbf{v}^{0}$. The contributions of large velocities to the solution and thus to the integrals in marginal tip density and flux are small. We therefore expect stable global in time solutions for data decaying exponentially fast without using that velocity cut-off.

\section{Conclusions}

We have addressed the numerical solution of a kinetic model of tumor driven angiogenesis, a process in which new blood vessels sprout from existing ones to supply the growing tumor with nutrients and oxygen. This model is derived from stochastic descriptions of the angiogenesis process and allows us to gain understanding on the evolution of the blood vessel network by means of asymptotic and numerical solutions. The ultimate goal would be to control the angiogenesis process and starve the tumor.

Employing reliable numerical discretizations is essential in these studies. However, the presence of nonlocal terms in time and velocity in the kinetic model, as well as the nonlinear coupling with a diffusion problem, render this task quite challenging. We have studied here a robust finite difference scheme of 
order-one which enjoys stability and positivity properties allowing to establish some convergence results. We have tested it tracking soliton-like solutions, for which an explicit asymptotic characterization is given. Employing ensemble averages we have compared predictions provided by the numerical solution of the kinetic model with predictions yielded by the original stochastic description. Good agreement is found in both cases.

Once the reliability of the scheme has been validated with these tests, we study numerically the influence of the velocity dependence in the definition of the flux of blood vessel tips, a point which is unclear from the modeling point of view and is relevant when establishing well posedness results for the kinetic model. Whereas the original derivation of the model uses a flux definition without velocity cut-offs, the theoretical well posedness results established so far require their presence. For exponentially decaying densities, our numerical comparisons show that the inclusion of velocities cut-offs does not really alter the solutions, and the model should still be well posed regardless of their presence. This is in agreement with the experimental observation that the velocities of the blood vessel tips remain within a certain range, achievable by cells.

Our scheme is effective, albeit with the drawback of being slow. This poses the problem of identifying adequate strategies to develop faster methods of higher order. The presence of terms that are nonlocal in time and the coupling with a diffusion equation through gradients render this task quite complex.

Acknowledgements. This work has been supported by MINECO grants No. MTM2014-56948-C2-1-P, MTM2017-84446-C2-1-R (AC, GD) and MTM201456948-C2-2-P, MTM2017-84446-C2-2-R (LB, MC, FT). The authors thank V. Capasso for discussions of angiogenesis, during a sabbatical visit supported by a Banco de Santander-Universidad Carlos III de Madrid chair of excellence.

\section{Appendix A. Soliton-like solutions}

This Appendix obtains reduced equations for the soliton-like solutions studied in the paper adapting to our flux definition a procedure developed in [5]. Here we work with different flux terms, of the form $j=\int|\mathbf{v}| p d \mathbf{v}$ and $j=$ $\int w(\mathbf{v}) p d \mathbf{v}$ where $w(\mathbf{v})$ is a weight including a velocity cut-off, though the final formulas happen to be similar. This kind of expansions might be the basis of higher order schemes.

\section{Appendix A.1. Derivation of the reduced equation for the marginal density}

We first find a reduced equation for the marginal density (5) in the limit of large friction, $\beta \rightarrow \infty$ [5]. The source terms in (1) (the two first terms on its right-hand side) favor velocities in a small neighborhood of $\mathbf{v}^{0}$, since such velocities are the only ones for which the birth term proportional to $\alpha(C) \delta_{\sigma_{v}}(\mathbf{v}-$ $\mathbf{v}^{0}$ ) [cf. Eq. (1)] may balance the anastomosis death term. A reduced equation 
for $\rho$ is obtained by the Chapman-Enskog method [5]. We rewrite (1) as

$$
\begin{aligned}
\mathcal{L} p & \equiv \beta \operatorname{div}_{\mathbf{v}}\left(\frac{1}{2} \nabla_{\mathbf{v}} p+\left(\mathbf{v}-\mathbf{v}^{0}\right) p\right)=\epsilon\left[\frac{\partial p}{\partial t}+\beta\left(\mathbf{F}-\mathbf{v}^{0}\right) \cdot \nabla_{\mathbf{v}} p\right. \\
& \left.+\mathbf{v} \cdot \nabla_{\mathbf{x}} p-\alpha p \delta_{\sigma_{v}}\left(\mathbf{v}-\mathbf{v}^{0}\right)+\Gamma p \int_{0}^{t} \rho(\mathbf{x}, s) d s\right] .
\end{aligned}
$$

A scaling parameter $\epsilon$ is included in the right hand side of this equation, which is considered small compared to the left hand side. After the computations that come next, we will restore $\epsilon=1$. Assuming $\epsilon \ll 1$, we expand $p$ in the asymptotic series

$$
p=p^{(0)}+\epsilon p^{(1)}+\epsilon^{2} p^{(2)}+\ldots
$$

For $\epsilon=0,(\mathrm{~A} .1)$ has a solution $p^{(0)}$ given by the right hand side of (60). Now, we assume that the marginal tip density obeys

$$
\frac{\partial \rho}{\partial t}=\mathcal{F}^{(0)}+\epsilon \mathcal{F}^{(1)}+\ldots,
$$

where the $\mathcal{F}^{(j)}$ are determined by solvability conditions to be derived next. Inserting (A.2) and (A.3) in (A.1) and equating the coefficients of like powers of $\epsilon$, we get the hierarchy of equations

$$
\begin{aligned}
& \mathcal{L} p^{(0)}=0 \\
& \mathcal{L} p^{(1)}=\frac{e^{-V^{2}}}{\pi}\left[\mathcal{F}^{(0)}+\mathbf{v} \cdot \nabla_{\mathbf{x}} \rho-2 \beta \mathbf{V} \cdot\left(\mathbf{F}-\mathbf{v}^{0}\right) \rho-\alpha \rho \delta_{\sigma_{v}}(\mathbf{V})\right. \\
& \left.\quad+\Gamma \rho \int_{0}^{t} \rho(\mathbf{x}, s) d s\right] \\
& \mathcal{L} p^{(2)}=\frac{e^{-V^{2}}}{\pi} \mathcal{F}^{(1)}+\mathbf{v} \cdot \nabla_{\mathbf{x}} p^{(1)}-2 \beta \mathbf{V} \cdot\left(\mathbf{F}-\mathbf{v}^{0}\right) p^{(1)}-\alpha p^{(1)} \delta_{\sigma_{v}}(\mathbf{V}) \\
& +\Gamma p^{(1)} \int_{0}^{t} \rho(\mathbf{x}, s) d s
\end{aligned}
$$

etc. Here $\mathbf{V}=\mathbf{v}-\mathbf{v}^{0}$ and $V=|\mathbf{V}|$. Inserting (A.2) into Eq. (5) defining $\rho$, we find

$$
\int p^{(0)} d \mathbf{v}=\rho, \quad \int p^{(j)} d \mathbf{v}=0, \quad j=1,2, \ldots
$$

The non-homogeneous linear equations (A.5) and (A.6) have bounded solutions when the conditions

$$
\int \mathcal{L} p^{(j)} d \mathbf{v}=0, \quad j=1,2, \ldots,
$$

hold, since the adjoint problem $\mathcal{L}^{\dagger} v=0$ has constant solutions. For (A.5), the solvability condition (A.8) implies

$$
\mathcal{F}^{(0)}=\frac{\alpha}{\pi} \rho-\mathbf{v}^{0} \cdot \nabla_{\mathbf{x}} \rho-\Gamma \rho \int_{0}^{t} \rho(\mathbf{x}, s) d s .
$$


For this choice of $\mathcal{F}^{(0)}$, the solution of (A.5) satisfying (A.7) is

$$
p^{(1)}=-\frac{e^{-V^{2}}}{\pi} \mathbf{V} \cdot\left[\nabla_{\mathbf{x}} \rho-2 \beta\left(\mathbf{F}-\mathbf{v}^{0}\right) \rho\right]+\frac{\alpha \rho}{2 \pi^{2}} e^{-V^{2}}\left[\int_{0}^{\infty} e^{-t} \ln t d t-\ln V^{2}\right]
$$

Inserting (A.10) into the solvability condition (A.8) for $j=2$ yields

$$
\mathcal{F}^{(1)}=\frac{1}{2 \beta} \Delta_{\mathbf{x}} \rho+\operatorname{div}_{\mathbf{x}}\left[\left(\mathbf{v}^{0}-\mathbf{F}\right) \rho\right]+\frac{\alpha^{2} \rho}{2 \pi^{2} \beta\left(1+\sigma_{v}^{2}\right)} \ln \left(1+\frac{1}{\sigma_{v}^{2}}\right) .
$$

Next, we place (A.9) and (A.11) in (A.3) and recall $\epsilon=1$, thereby obtaining the Smoluchowski-type equation

$$
\begin{array}{r}
\frac{\partial \rho}{\partial t}+\operatorname{div}_{\mathbf{x}}(\mathbf{F} \rho)-\frac{1}{2 \beta} \Delta_{\mathbf{x}} \rho=\mu \rho-\Gamma \rho \int_{0}^{t} \rho(\mathbf{x}, s) d s, \\
\mu=\frac{\alpha}{\pi}\left[1+\frac{\alpha}{2 \pi \beta\left(1+\sigma_{v}^{2}\right)} \ln \left(1+\frac{1}{\sigma_{v}^{2}}\right)\right] .
\end{array}
$$

Appendix A.2. Derivation of the soliton formula (63)

Let us define

$$
R(\mathbf{x}, t)=\int_{0}^{t} \rho(\mathbf{x}, s) d s
$$

Ignoring diffusion in (A.12) we get

$$
\frac{\partial^{2} R}{\partial t^{2}}+\operatorname{div}_{\mathbf{x}}\left(\mathbf{F} \frac{\partial R}{\partial t}\right)=\mu \frac{\partial R}{\partial t}-\Gamma R \frac{\partial R}{\partial t} .
$$

The coefficients $\chi$ and $\kappa$ in (2) are very small [3]. Therefore, the TAF concentration varies very slowly compared to the marginal tip density. We additionally assume that the TAF gradient is oriented along the $x_{1}$ axis and that the initial TAF concentration varies on a larger spatial scale than the soliton size, which furnishes a good approximation [3]. As a result, $\mathbf{F}$ and $\mu$ are nearly constant. We then look for a solution of the form

$$
R(\mathbf{x}, t)=R(\xi), \quad \xi=x_{1}-c t,
$$

for (A.15). This yields the ordinary differential equation

$$
\left(c-F_{1}\right) \frac{\partial^{2} R}{\partial \xi^{2}}+(\mu-\Gamma R) \frac{\partial R}{\partial \xi}=0,
$$

with $\mathbf{F}=\left(F_{1}, F_{2}\right)$. Integrating identity (A.17) once, we find

$$
\left(c-F_{1}\right) \frac{2}{\Gamma} \frac{\partial R}{\partial \xi}=\rho^{2}-2 \frac{\mu}{\Gamma} R-\frac{2 K}{\Gamma}
$$


for a constant $K$. Setting $R=\frac{\mu}{\Gamma}+\nu \tanh (\lambda \xi)$, we obtain $\nu^{2}=\frac{\mu^{2}+2 K \Gamma}{\Gamma^{2}}$ and $2 \nu \lambda\left(c-F_{1}\right) / \Gamma=-\nu^{2}$, thereby concluding

$$
R=\frac{\mu}{\Gamma}-\frac{\sqrt{2 K \Gamma+\mu^{2}}}{\Gamma} \tanh \left[\frac{\sqrt{2 K \Gamma+\mu^{2}}}{2\left(c-F_{1}\right)}\left(\xi-\xi_{0}\right)\right],
$$

where $\xi_{0}$ is a constant of integration. Hence, $\rho=\frac{\partial R}{\partial t}=-c \frac{\partial R}{\partial \xi}$ provides

$$
\rho=\frac{\left(2 K \Gamma+\mu^{2}\right) c}{2 \Gamma\left(c-F_{1}\right)} \operatorname{sech}^{2}\left[\frac{\sqrt{2 K \Gamma+\mu^{2}}}{2\left(c-F_{1}\right)}\left(x_{1}-c t-\xi_{0}\right)\right],
$$

which is $(63)$.

Appendix A.3. Derivation of the collective coordinates equations (65)-(66)

Summarizing, we can write the soliton solution (63) as

$$
\begin{aligned}
\rho_{s} & =\frac{\left(2 K \Gamma+\mu^{2}\right) c}{2 \Gamma\left(c-F_{1}\right)} \operatorname{sech}^{2} s, \\
s & =\frac{\sqrt{2 K \Gamma+\mu^{2}}}{2\left(c-F_{1}\right)} \xi, \quad \xi=x_{1}-X(t), \\
\dot{X} & =\frac{d X}{d t}=c .
\end{aligned}
$$

The functions $X(t), c(t)$ and $K(t)$ are time-dependent collective coordinates characterizing the soliton. They are supposed to vary slowly due to the small diffusivity in (A.12) and to the slow variation of the TAF concentration. Then, the marginal tip density is represented by a soliton that moves and changes shape slowly, in accordance with the variations of its collective coordinates. Note that the function $\rho_{s}$ depends on $\xi$ and also on $\mathbf{x}$ and $t$ through $C(t, \mathbf{x})$,

$$
\rho_{s}=\rho_{s}\left(\xi ; K, c, \mu(C), F_{1}\left(C, \frac{\partial C}{\partial x_{1}}\right)\right) .
$$

Next, we assume that the space and time variations of $C$, which are present when $\rho_{s}$ is differentiated with respect to $x_{1}$ or $t$, generate terms that are small compared with $\partial \rho_{s} / \partial \xi$. These terms are neglected, therefore the different definitions of the flux in (2) do not affect the equations for the collective coordinates. We shall consider that $\mu(C)$ is almost constant, ignore $\partial C / \partial t$ because the TAF concentration varies slowly (the dimensionless coefficients $\kappa$ and $\chi_{1}$ in the TAF equation (61) are very small) and ignore $\partial^{2} \rho_{s} / \partial i \partial j$, where $i, j=K, F_{1}$. More general situations are discussed in [5]. Inserting (A.21) and (A.22) into (A.12) we obtain

$$
\begin{array}{r}
\left(F_{1}-\dot{X}\right) \frac{\partial \rho_{s}}{\partial \xi}+\frac{\partial \rho_{s}}{\partial K} \dot{K}+\frac{\partial \rho_{s}}{\partial c} \dot{c}+\rho_{s} \operatorname{div}_{\mathbf{x}} \mathbf{F}+\frac{\partial \rho_{s}}{\partial F_{1}}\left(\frac{\partial F_{1}}{\partial t}+\mathbf{F} \cdot \nabla_{\mathbf{x}} F_{1}\right) \\
-\frac{1}{2 \beta}\left(\frac{\partial^{2} \rho_{s}}{\partial \xi^{2}}+2 \frac{\partial^{2} \rho_{s}}{\partial \xi \partial F_{1}} \frac{\partial F_{1}}{\partial x_{1}}+\frac{\partial \rho_{s}}{\partial F_{1}} \Delta_{\mathbf{x}} F_{1}\right)=\mu \rho_{s}-\Gamma \rho_{s} \int_{0}^{t} \rho_{s} d t
\end{array}
$$


Eq. (A.12) with $1 / \beta=0$ and $\mathbf{F}$ constant has the soliton-like solution (A.21)(A.22). Using this fact and (A.23), identity (A.25) becomes

$$
\begin{aligned}
& \frac{\partial \rho_{s}}{\partial K} \dot{K}+\frac{\partial \rho_{s}}{\partial c} \dot{c}=\mathcal{A} \\
& \mathcal{A}=\frac{1}{2 \beta} \frac{\partial^{2} \rho_{s}}{\partial \xi^{2}}-\rho_{s} \operatorname{div}_{\mathbf{x}} \mathbf{F}-\frac{\partial \rho_{s}}{\partial F_{1}}\left[\mathbf{F} \cdot \nabla_{\mathbf{x}} F_{1}-\frac{1}{2 \beta} \Delta_{\mathbf{x}} F_{1}\right]+\frac{1}{\beta} \frac{\partial^{2} \rho_{s}}{\partial \xi \partial F_{1}} \frac{\partial F_{1}}{\partial x_{1}} .
\end{aligned}
$$

Now, we derive collective coordinate equations (CCEs) for $K$ and $c$. As the lump-like soliton advances along the $x_{1}$ axis, we set $x_{2}=0$ to locate the position of its maximum. On the $x_{1}$ axis, the profile is given by (A.21)-(A.22). We multiply (A.26) by $\partial \rho_{s} / \partial K$ and integrate over $x_{1}$. Then, we consider a fully formed soliton far from the tumor and the primary vessel. Due to exponential decay for $|\xi| \gg 1$, this soliton is localized on some interval $(-\mathcal{L} / 2, \mathcal{L} / 2)$. The coefficients in the formulas (A.21)-(A.22) and the coefficients in equation (A.26) depend on the TAF concentration at $x_{2}=0$. Thus, they are functions of space and time and are integrated over $x_{1}$. Moreover, the TAF varies slowly on the support of the soliton. Therefore we approximate the integrals over $x_{1}$ by

$$
\int_{\mathcal{I}} G\left(\rho_{s}\left(\xi ; x_{1}, t\right), x_{1}\right) d x_{1} \approx \frac{1}{\mathcal{L}} \int_{\mathcal{I}}\left(\int_{-\mathcal{L} / 2}^{\mathcal{L} / 2} G\left(\rho_{s}\left(\xi ; x_{1}, t\right), x_{1}\right) d \xi\right) d x_{1},
$$

see [5] for details. The integration interval $\mathcal{I}$ should be large enough to contain most of the soliton, of width $\mathcal{L}$. In view of this, the CCEs hold only after the initial soliton formation stage. Near the tumor, the boundary condition alters the soliton and we should exclude an interval about $x_{1}=1$ from $\mathcal{I}$. In a similar way, we multiply (A.26) by $\partial \rho_{s} / \partial c$ and integrate over $x_{1}$. The two resulting formulas define $\dot{K}$ and $\dot{c}$ as fractions. The factors $1 / \mathcal{L}$ cancel out from their denominators and numerators. Since the soliton tails decay exponentially to zero, we set $\mathcal{L} \rightarrow \infty$ and find the following CCEs

$$
\begin{gathered}
\dot{K}=\frac{\int_{-\infty}^{\infty} \frac{\partial \rho_{s}}{\partial K} \mathcal{A} d \xi \int_{-\infty}^{\infty}\left(\frac{\partial \rho_{s}}{\partial c}\right)^{2} d \xi-\int_{-\infty}^{\infty} \frac{\partial \rho_{s}}{\partial c} \mathcal{A} d \xi \int_{-\infty}^{\infty} \frac{\partial \rho_{s}}{\partial K} \frac{\partial \rho_{s}}{\partial c} d \xi}{\int_{-\infty}^{\infty}\left(\frac{\partial \rho_{s}}{\partial K}\right)^{2} d \xi \int_{-\infty}^{\infty}\left(\frac{\partial \rho_{s}}{\partial c}\right)^{2} d \xi-\left(\int_{-\infty}^{\infty} \frac{\partial \rho_{s}}{\partial c} \frac{\partial \rho_{s}}{\partial K} d \xi\right)^{2}} \\
\dot{c}=\frac{\int_{-\infty}^{\infty} \frac{\partial \rho_{s}}{\partial c} \mathcal{A} d \xi \int_{-\infty}^{\infty}\left(\frac{\partial \rho_{s}}{\partial K}\right)^{2} d \xi-\int_{-\infty}^{\infty} \frac{\partial \rho_{s}}{\partial K} \mathcal{A} d \xi \int_{-\infty}^{\infty} \frac{\partial \rho_{s}}{\partial K} \frac{\partial \rho_{s}}{\partial c} d \xi}{\int_{-\infty}^{\infty}\left(\frac{\partial \rho_{s}}{\partial K}\right)^{2} d \xi \int_{-\infty}^{\infty}\left(\frac{\partial \rho_{s}}{\partial c}\right)^{2} d \xi-\left(\int_{-\infty}^{\infty} \frac{\partial \rho_{s}}{\partial c} \frac{\partial \rho_{s}}{\partial K} d \xi\right)^{2}}
\end{gathered}
$$

In these equations, all the terms changing slowly in space have been averaged over the interval $\mathcal{I}$. The last term in (A.27) being odd in $\xi$ does not affect the integrals in (A.29) and (A.30) while the remaining terms in (A.27) are even in $\xi$ and add to them. The integrals in (A.29) and (A.30) are calculated in [5]. The final CCEs are (65) and (66).

\section{References}


[1] M. Asadzadeh, P. Kowalczyk, Convergence analysis of the streamline diffusion and discontinuous Galerkin methods for the Vlasov-Fokker-Planck system, Numer. Methods Partial Differ. Equat. 21, 472-495, 2005

[2] M. Asadzadeh, A. Sopasakis, Convergence of a hp-streamline diffusion scheme for Vlasov-Fokker-Planck system, Math. Models Methods Appl. Sci. 17 (8) 1159-1182, 2007

[3] L.L. Bonilla, V. Capasso, M. Alvaro, M. Carretero, Hybrid modeling of tumor-induced angiogenesis, Physical Review E 90, 062716, 2014

[4] L.L. Bonilla, M. Carretero, F. Terragni, B. Birnir, Soliton driven angiogenesis, Scientific Reports 6, 31296, 2016

[5] L.L. Bonilla, M. Carretero, F. Terragni, Solitonlike attractor for blood vessel tip density in angiogenesis, Physical Review E 94, 062415, 2016

[6] L.L. Bonilla, M. Carretero, F. Terragni, Ensemble averages, soliton dynamics and influence of haptotaxis in a model of tumor-induced angiogenesis, Entropy 19, 209, 2017

[7] C. Buet, S. Cordier, P. Degond, M. Lemou, Fast algorithms for numerical, conservative, and entropy approximations of the Fokker-Planck-Landau equation, Journal of Computational Physics 133(2), 310-322, 1997

[8] V. Capasso and D. Morale, Stochastic modelling of tumour-induced angiogenesis, J. Math. Biol. 58, 219-233, 2009

[9] V. Capasso, D. Morale, G. Facchetti, Randomness in self-organized phenomena. A case study: Retinal angiogenesis, BioSystems 112, 292-297, 2013.

[10] V. Capasso, F. Flandoli, On the mean field approximation of a stochastic model of tumor-induced angiogenesis. arXiv:1708.03830.

[11] P.F. Carmeliet, Angiogenesis in life, disease and medicine, Nature 438, 932-936, 2005

[12] P.F. Carmeliet and R. K. Jain, Molecular mechanisms and clinical applications of angiogenesis, Nature 473, 298-307, 2011

[13] A. Carpio, G. Duro, Well posedness of an integrodifferential kinetic model of Fokker-Planck type for angiogenesis, Nonlinear Analysis: Real World Applications 30, 184-212, 2016

[14] A. Carpio, G. Duro, M. Negreanu, Constructing solutions for a kinetic model of angiogenesis in annular domains, Applied Mathematical Modelling 45, 303-322, 2017

[15] L. Chacón, D.C. Barnes, D.A. Knoll, G.H. Miley, An implicit energyconservative 2D Fokker- Planck Algorithm: I. Difference scheme, Journal of Computational Physics, 157(2), 618-653, 2000 
[16] C.Z. Cheng, G. Knorr, The integration of the Vlasov equation in configuration space, Journal of Computational Physics 22, 330-351, 1976

[17] G.H. Cottet, P.A. Raviart, Particle methods for the one-dimensional Vlasov-Poisson equations, SIAM J. Numer. Anal. 21, 52-76, 1984

[18] P. Degond, Global existence of smooth solutions for the Vlasov-FokkerPlanck equation in 1 and 2 space dimensions, Ann. Sci. Ec. Norm. Super. 19 (4), 519-542, 1986.

[19] P. Degond, L. Pareschi, G. Russo, Modeling and computational methods for kinetic equations, Springer Science \& Business Media, 2004

[20] L. Einkemmer, Splitting methods for Vlasov-Poisson and Vlasov-Maxwell equations, PhD Thesis, University of Innsbruck 2014

[21] F. Filbet, E. Sonnendrücker, P. Bertrand, Conservative numerical schemes for the Vlasov equation, Journal of Computational Physics 172, 166-187, 2001

[22] F. Filbet, E. Sonnendrücker, Comparison of Eulerian Vlasov solvers, Computer Physics Communications 150(3), 247-266, 2003

[23] J. Folkman, Tumor angiogenesis. Therapeutic implications. N Engl J Med 285, 1182-1186, 1971

[24] C. W. Gardiner, Stochastic methods. A handbook for the natural and social sciences. 4th ed. Springer, Berlin 2010

[25] K.J. Havlak, H.D. Victory Jr., The numerical analysis of random particle methods applied to Vlasov-Poisson-Fokker-Planck kinetic equations, SIAM J. Numer. Anal. 33, 291-317, 1996

[26] K.J. Havlak, H.D. Victory Jr., On deterministic particle methods for solving Vlasov-Poisson-Fokker-Planck systems, SIAM J. Numer. Anal. 35(4) 1473-1519, 1998

[27] T.A.M. Heck, M.M. Vaeyens, H. Van Oosterwyck, Computational models of sprouting angiogenesis and cell migration: towards multiscale mechanochemical models of angiogenesis, Math Model Nat Phenom 10, 09735348, 2015

[28] R.J. Kingham, A.R. Bell, An implicit Vlasov-Fokker-Planck code to model non-local electron transport in 2-D with magnetic fields, Journal of Computational Physics 194(1), 1-34, 2004

[29] A. Marquina, Diffusion front capturing schemes for a class of Fokker-Planck equations: Application to the relativistic heat equation, Journal of Computational Physics 229(7), 2659-2674, 2010 
[30] F. Milde, M. Bergdorf, P. Koumoutsakos, A hybrid model for threedimensional simulations of sprouting angiogenesis, Biophys J. 95, 3146-3160, 2008

[31] O.L. Mueller, C. Pares, E.F. Toro, Well-balanced high-order numerical schemes for onedimensional blood flow in vessels with varying mechanical properties, Journal of Computational Physics 242, 53-85, 2013

[32] S.M. Peirce, Computational and mathematical modeling of angiogenesis, Microcirculation 15(8), 739-751, 2008

[33] J.M. Qiu, A. Christlieb, A conservative high order semi-Lagrangian WENO method for the Vlasov equation Journal of Computational Physics 229(4), $1130-1149,2010$

[34] M. Scianna, L. Munaron, and L. Preziosi, A multiscale hybrid approach for vasculogenesis and related potential blocking therapies, Prog. Biophys. Mol. Biol. 106, 450-462, 2011

[35] M. Scianna, C.G. Bell, L. Preziosi, A review of mathematical models for the formation of vascular networks, J. Theor. Biol. 333, 174-209, 2013

[36] D. Seal, Discontinuous Galerkin methods for Vlasov models of plasma, PhD Thesis, University of Wisconsin-Madison, 2012

[37] B. Sepehrian, M.K. Radpoor Numerical solution of non-linear FokkerPlanck equation using finite differences method and the cubic spline functions, Applied Mathematics and Computation, 262, 187-190, 2015

[38] E. Sonnendrücker, J. Roche, P. Bertrand, A. Ghizzo, The semi-Lagrangian method for the numerical resolution of Vlasov equations, Journal of Computational Physics 149(2), 201-220, 1999

[39] S. Sun, M.F. Wheeler, M. Obeyesekere, Ch. Patrick Jr., Multiscale angiogenesis modeling using mixed finite element methods, Multiscale Model. Simul. 4(4), 1137-1167, 2005

[40] K. R. Swanson, R. C. Rockne, J. Claridge, M. A. Chaplain, E. C. Alvord Jr., and A. R. A. Anderson, Quantifying the role of angiogenesis in malignant progression of gliomas: in silico modeling integrates imaging and histology, Cancer Res 71, 7366-7375, 2011

[41] W.T. Taitano, L. Chacón, A.N. Simakov, K. Molvig, A mass, momentum, and energy conserving, fully implicit, scalable algorithm for the multidimensional, multi-species Rosenbluth-Fokker-Planck equation, Journal of Computational Physics 297(1), 357-380, 2015

[42] F. Terragni, M. Carretero, V. Capasso, L. L. Bonilla, Stochastic model of tumor-induced angiogenesis: Ensemble averages and deterministic equations, Physical Review E 93, 022413, 2016 
[43] B. Wang, G. H. Miller, P. Colella, A particle-in-cell method with adaptive phase-space remapping for kinetic plasmas, SIAM J. Sci. Comput., 33(6), 3509-3537, 2011

[44] S. Wollman, E. Ozizmir, Numerical approximation of the Vlasov-PoissonFokker-Planck system in two dimensions, Journal of Computational Physics $228,6629-6669,2009$

[45] L. Yadav, N. Puri, V. Rastogi, P. Satpute, V. Sharma, Tumour angiogenesis and angiogenic inhibitors: a review, J. Clin. Diagn. Res. 9, XE01-XE05, 2015 